TRANSACTIONS OF THE

AMERICAN MATHEMATICAL SOCIETY

Volume 359, Number 8, August 2007, Pages 3769-3789

S 0002-9947(07)04277-8

Article electronically published on March 20, 2007

\title{
RIEMANNIAN FLAG MANIFOLDS WITH HOMOGENEOUS GEODESICS
}

\author{
DMITRI ALEKSEEVSKY AND ANDREAS ARVANITOYEORGOS
}

\begin{abstract}
A geodesic in a Riemannian homogeneous manifold $(M=G / K, g)$ is called a homogeneous geodesic if it is an orbit of a one-parameter subgroup of the Lie group $G$. We investigate $G$-invariant metrics with homogeneous geodesics (i.e., such that all geodesics are homogeneous) when $M=G / K$ is a flag manifold, that is, an adjoint orbit of a compact semisimple Lie group $G$. We use an important invariant of a flag manifold $M=G / K$, its $T$-root system, to give a simple necessary condition that $M$ admits a non-standard $G$-invariant metric with homogeneous geodesics. Hence, the problem reduces substantially to the study of a short list of prospective flag manifolds. A common feature of these spaces is that their isotropy representation has two irreducible components. We prove that among all flag manifolds $M=G / K$ of a simple Lie group $G$, only the manifold $\operatorname{Com}\left(\mathbb{R}^{2 \ell+2}\right)=S O(2 \ell+1) / U(\ell)$ of complex structures in $\mathbb{R}^{2 \ell+2}$, and the complex projective space $\mathbb{C} P^{2 \ell-1}=S p(\ell) / U(1) \cdot S p(\ell-1)$ admit a non-naturally reductive invariant metric with homogeneous geodesics. In all other cases the only $G$-invariant metric with homogeneous geodesics is the metric which is homothetic to the standard metric (i.e., the metric associated to the negative of the Killing form of the Lie algebra $\mathfrak{g}$ of $G$ ). According to F. Podestà and G.Thorbergsson (2003), these manifolds are the only nonHermitian symmetric flag manifolds with coisotropic action of the stabilizer.
\end{abstract}

\section{Introduction}

A Riemannian manifold $(M, g)$ is called homogeneous if it admits a transitive connected Lie group $G$ of isometries. Then $M$ can be viewed as a coset space $G / K$ with a $G$-invariant metric, where $K$ is the isotropy subgroup of some point in $M$. A geodesic $\gamma(t)$ through the origin $o=e K$ is called a homogeneous geodesic if it is an orbit of a one-parameter subgroup of $G$, i.e.,

$$
\gamma(t)=(\exp t X) \cdot o
$$

where $X$ is a non-zero vector in the Lie algebra $\mathfrak{g}$ of $G$. Homogeneous geodesics were originally studied quite a long time ago by several authors such as R. Hermann, B. Kostant, and E.B. Vinberg to name a few. In particular, in Kos1 and Vin a simple algebraic condition was found so that the orbit (1) is a geodesic.

Homogeneous geodesics in a Lie group were studied by V.V. Kajzer in Kaj] where he proved that a Lie group $G$ with a left-invariant metric has at least one

Received by the editors June 23, 2005.

2000 Mathematics Subject Classification. Primary 53C22, 53C30; Secondary 14M15.

Key words and phrases. Homogeneous Riemannian manifolds, flag manifolds, homogeneous geodesics, g.o. spaces, coisotropic actions.

The first author was supported by Grant Luverhulme trust, EM/9/2005/0069.

(C)2007 American Mathematical Society Reverts to public domain 28 years from publication 
homogeneous geodesic through the identity. In $[\mathrm{Sz}]$ J. Szenthe proved that if a compact connected and semisimple Lie group has rank greater than 1, then for every left-invariant metric there are infinitely many homogeneous geodesics through the identity element. In $\mathrm{Ma}$ R. A. Marinosci investigated the set of all homogeneous geodesics in a 3-dimensional Lie group. A generalization of Kajzer's result was obtained by O. Kowalski and J. Szenthe ( $\overline{\mathrm{Ko}-\mathrm{Sz}}$ ) who proved that every Riemannian homogenous manifold admits at least one homogeneous geodesic through each point.

Besides their mathematical significance, homogeneous geodesics have important applications in mechanics. The equation of motion of many systems of classical mechanics reduces to the geodesic equation in an appropriate Riemannian manifold $M$. Homogeneous geodesics of $M$ correspond to "relative equilibriums" of the corresponding system (see V.I. Arnold [Arn] ). There is a vast literature in mechanics devoted to the investigation of relative equilibria (e.g., Ga-Hu-Wi] and relevant references).

Riemannian homogeneous spaces of special importance are spaces where all geodesics are homogeneous. In fact these spaces are examples of a wider important class of Riemmanian manifolds, the D'Atri spaces, where each local geodesic symmetry preserves the Riemannian volume element (cf. Ko-Pr-Va]).

Riemannian homogeneous spaces with all geodesics homogeneous are also known as g.o. spaces (from "geodesic orbit"). The terminology was introduced by O. Kowalski and L. Vanhecke in Ko-Va, who initiated a systematic study of such spaces. Examples of such spaces are the symmetric spaces, the weakly symmetric spaces and the naturally reductive spaces. Weakly symmetric spaces are Riemannian manifolds $(M, g)$ such that for any two points $p, q \in M$ there exists an isometry which interchanges $p$ and $q$. Naturally reductive spaces are Riemannian manifolds $(M, g)$ whose metric $g$ is induced by an $\mathrm{Ad}^{G}$-invariant non-degenerate symmetric bilinear form on the Lie algebra $\mathfrak{g}$ of some transitive group $G$ of isometries. The class of g.o. spaces is larger than the naturally reductive spaces. In fact, A. Kaplan ( $\mathrm{Ka}$ ) gave the first example of a g.o. space which is not naturally reductive. Since then, there was a lot of research on homogeneous spaces where all geodesics are homogeneous. In $\mathrm{Ko}-\mathrm{Va}$, O. Kowalski and L. Vanhecke classified all g.o. spaces in dimension $\leq 6$. In $\mathrm{Go}$, C. Gordon reduced the classification of g.o. spaces $M$ to three special cases: (a) $M$ is a nilmanifold (i.e., a nilpotent Lie group with leftinvariant Riemannian metric), (b) $M$ is compact, and (c) $M$ admits a transitive non-compact semisimple Lie group of isometries. She described g.o. spaces for case (a). Another approach for the description of g.o. spaces was proposed by O. Kowalski, S. Ž. Nikčević and Z. Vlašek [Ko-Ni] and Ko-Ni-Vl], as well as by Z. Dušek [Du1] and Du2]. Recently, Z. Dušek, O. Kowalski and S. Ž. Nikčević ([Du-Ko-Ni]) gave examples of g.o. spaces in dimension 7 whose full groups of isometries are not semisimple. Finally, H. Tamaru ([Ta $)$ classified homogeneous g.o. spaces which are fibered over irreducible symmetric spaces.

The general problem of classification of compact homogeneous Riemannian manifolds $(M=G / K, g)$ with homogeneous geodesics remains open.

The aim of this paper is to give a complete classification of Riemannian flag manifolds with homogeneous geodesics (i.e., such that all geodesics are homogeneous). Recall that a flag manifold is an adjoint orbit $M=\operatorname{Ad}_{G} w=G / K(w \in \operatorname{Lie}(G))$ of 
a compact semisimple Lie group $G$, and a flag manifold equipped with an invariant Riemannian metric is called a Riemannian flag manifold.

Let $M=G / K$ be a flag manifold. If the group $G$ acts effectively on $M$, it has no center and is a direct product

$$
G=G_{1} \times \cdots \times G_{k}
$$

of simple factors. Moreover, if $g$ is an invariant metric on $M$, then the Riemannian flag manifold $(M=G / K, g)$ is a Riemannian direct product

$$
M=G / K=G_{1} / K_{1} \times \cdots \times G_{k} / K_{k}
$$

of Riemannian flag manifolds $\left(M_{i}=G_{i} / K_{i}, g_{i}\right)=\left.g\right|_{M_{i}}$, where $K_{i}=K \cap G_{i}$.

The manifold $(M, g)$ has homogeneous geodesics if and only if each factor $\left(M_{i}, g_{i}\right)$ has homogeneous geodesics. This reduces the problem of the description of $G$ invariant metrics with homogeneous geodesics on a flag manifold $M=G / K$ to the case when the group $G$ is simple.

Flag manifolds $M=G / K$ of a simple Lie group $G$ can be classified in terms of their painted Dynkin diagrams (cf. Section 3). In particular, for the exceptional Lie groups $G_{2}, F_{4}, E_{6}, E_{7}$, and $E_{8}$ there are $3,11,16,31$, and 40 non-equivalent flag manifolds, respectively. An important invariant of a flag manifold $M=G / K$ is the system of $T$-roots $R_{T}$. It is defined as the restriction of the root system $R$ of the corresponding Lie algebra $\mathfrak{g}$ to the center $\mathfrak{t}$ of the (stability) subalgebra $\mathfrak{k}$ of $K$. We define the notion of a connected $T$-root system as follows: The $T$-root system is called connected if any two $T$-roots can be connected by a chain of $T$-roots whose sum or difference is also a $T$-root. We prove the following (cf. Theorem 7 ):

Theorem I. If the set of $T$-roots of a flag manifold $M=G / K$ is connected, then the only invariant metric with homogeneous geodesics on $M$ is the metric homothetic to the standard metric of $M$.

We describe flag manifolds of a simple Lie group with a non-connected system of $T$-roots. It turns out (cf. Propositions 9 and 10) that there are three infinite series of such flag manifolds of a classical Lie group (namely the spaces $B(\ell-m, m)=$ $S O(2 \ell+1) / U(\ell-m) \cdot S O(2 m+1), C(\ell-m, m)=S p(\ell) / U(\ell-m) \cdot S p(m)$, and $D(\ell-m, m)=S O(2 \ell) / U(\ell-m) \cdot S O(2 m))$ and 10 flag manifolds of an exceptional Lie group. Note that $B(\ell-m, m)$ (respectively $D(\ell-m, m)$ ) can be identified with the manifold of CR structures of codimension $2 m+1$ (respectively $2 m$ ) in $\mathbb{R}^{2 \ell+1}$ $\left(\right.$ respectively $\left.\mathbb{R}^{2 \ell}\right)$. A posteriori, we get the following characterization of these flag manifolds.

Let $G$ be a compact simple Lie group, $R$ the root system of the corresponding complex Lie algebra $\mathfrak{g}^{\mathbb{C}}$ with respect to a Cartan subalgebra $\mathfrak{h}^{\mathbb{C}}$ and $\Pi \subset R$ be a system of simple roots. For each simple root $\alpha \in \Pi$ we associate a flag manifold $M_{\alpha}=G / K_{\alpha}$ where $K_{\alpha}$ is the compact subgroup of $G$ associated with the complex subalgebra $\mathfrak{k}_{\alpha}$ of $\mathfrak{g}^{\mathbb{C}}$, generated by $\mathfrak{h}^{\mathbb{C}}$ and the root vectors $\left\{E_{\beta}: \beta \in \Pi \backslash\{\alpha\}\right\}$.

Theorem II. Let $M=G / K$ be a flag manifold of a simple Lie group $G$ with $T$-root system $R_{T}$. Then the following conditions are equivalent:

(1) $R_{T}$ is not connected,

(2) $R_{T}=\{ \pm \xi, \pm 2 \xi\}$ for some linear form $\xi \in \mathfrak{t}^{*}$,

(3) the isotropy representation of $K$ has two irreducible components,

(4) the flag manifold $M$ is associated with a simple root $\alpha$ with Dynkin mark 2, i.e., $M=M_{\alpha}=G / K_{\alpha}$. 
A simple root $\alpha \in \Pi$ defines a gradation

$$
\mathfrak{g}^{\mathbb{C}}=\sum_{i} \mathfrak{g}_{i}, \quad\left[\mathfrak{g}_{i}, \mathfrak{g}_{j}\right] \subset \mathfrak{g}_{i+j}
$$

of the complex Lie algebra $\mathfrak{g}^{\mathbb{C}}$ defined by the conditions

$$
\operatorname{deg} \mathfrak{h}^{\mathbb{C}}=0, \operatorname{deg} E_{\beta}=0, \text { for all } \beta \in \Pi \backslash \alpha, \operatorname{deg} E_{\alpha}=1 .
$$

The even and odd parts of a gradation determine a symmetric decomposition

$$
\mathfrak{g}^{\mathbb{C}}=\mathfrak{g}_{\mathrm{e} v}+\mathfrak{g}_{\mathrm{o} d d}=\sum \mathfrak{g}_{2 i}+\sum \mathfrak{g}_{2 i+1}
$$

of the Lie algebra $\mathfrak{g}^{\mathbb{C}}$ and hence a compact symmetric space $G / G_{\mathrm{ev}}$. The flag manifold $M_{\alpha}=G / K_{\alpha}$ is fibered over the corresponding compact symmetric space $G / G_{\mathrm{e} v}$ with fiber $G_{\mathrm{e} v} / K_{\alpha}$. If the Dynkin mark of $\alpha$ is 2 , the fiber is isotropy irreducible and any invariant metric on $M_{\alpha}$ (up to a scaling) is obtained from the standard metric $g_{1}$ by scaling its vertical part by $\lambda>0$. We denote this metric by $g_{\lambda}$.

In [Ta] H. Tamaru classified all such metrics which have homogeneous geodesics, for any homogeneous Riemannian manifold which is fibered over an irreducible symmetric space. His classification shows that the only flag manifolds $M_{\alpha}$ which admit a non-standard metric $g_{\lambda}, \lambda \neq 1$ with homogeneous geodesics are the manifolds $B(\ell, 0)=S O(2 \ell+1) / U(\ell)$ and $C(1, \ell-1)=S p(\ell) / U(1) \cdot S p(\ell-1)$. The classification by D.N. Akhiezer and E.B. Vinberg Ak-Vin of compact weakly symmetric spaces shows that they are weakly symmetric. Furthermore, a result by A.L. Onishchik $\mathrm{On}$ allows us to determine the full isometry group of any invariant metric $g_{\lambda}$ on these manifolds, hence the following final result.

Theorem III. The only flag manifolds $M=G / K$ of a simple Lie group $G$ which admit an invariant metric with homogeneous geodesics, not homothetic to the standard metric, are the manifolds $\left.B(\ell, 0)=\operatorname{Com}\left(\mathbb{R}^{2 \ell+2}\right)=S O(2 \ell+1) / U(\ell)\right)$ of complex structures in $\mathbb{R}^{2 \ell+2}$ and the complex projective space $C(1, \ell-1)=\mathbb{C} P^{2 \ell-2}=$ $S p(\ell) / U(1) \cdot S p(\ell-1)$. These manifolds admit a one-parameter family $g_{\lambda}, \lambda>0$ of invariant metrics (up to a scaling). All these metrics have homogeneous geodesics and are weakly symmetric. The metric $g_{1}$ is the standard metric. It has the full connected isometry group $S O(2 \ell+2)$ (respectively $S U(2 \ell-1)$ ) and is the standard metric of the symmetric space $\operatorname{Com}\left(\mathbb{R}^{2 \ell+2}\right)=S O(2 \ell+2) / U(\ell+1)$ (respectively $\left.\mathbb{C} P^{2 \ell-2}=S U(2 \ell-1) / U(2 \ell-2)\right)$. All the other metrics $g_{\lambda}, \lambda \neq 1$ have the full connected isometry group $S O(2 \ell+1)$ (respectively $S p(\ell)$ ). In particular, the corresponding spaces are not naturally reductive as Riemannian manifolds.

Note that for $\ell=2$ we obtain $S p(2) / U(1) \cdot S p(1) \cong S O(5) / U(2)$, which is the 6 -dimensional not naturally reductive g.o. space of O. Kowalski and L. Vanhecke Ko-Va.

We also remark that the flag manifolds of Theorem III appear in a recent work of F. Podestà and G. Thorbergsson ( $[\mathrm{Po}-\mathrm{Th}])$ as the only non-Hermitian symmetric flag manifolds $M=G / K$ such that the action of $K$ on $M$ is coisotropic.

The paper is organized as follows: In Section 2 we give an algebraic necessary and sufficient condition for a homogeneous Riemannian manifold to have homogeneous geodesics. In Section 3 we summarize various facts about flag manifolds (Lie-theoretic description, $T$-roots, isotropy representation, invariant metrics, and painted Dynkin diagrams). We give the complete list of flag manifolds $G / K$ of a 
simple Lie group $G$ and describe $G$-invariant metrics in terms of $T$-roots. In Section 4 we prove that a flag manifold with a non-standard metric with homogeneous geodesics has a non-connected $T$-root system. In Sections 5 and 6 we classify all flag manifolds $M=G / K$ of a simple Lie group $G$ with a non-connected $T$-root system. We determine which of these manifolds have non-standard metrics with homogeneous geodesics in Section 7, by using Tamaru's results.

\section{Homogeneous geodesics in Riemannian homogeneous spaces}

A Riemannian manifold $(M, g)$ is called homogeneous if it admits a transitive connected Lie group $G$ of isometries. Such a manifold may be identified with the coset space $G / K$, where $K$ is the isotropy subgroup of a point $o=e K \in M$. We assume that $G$ is semisimple and compact and that it acts effectively on $G / K$. If $\operatorname{Ad}_{G}: G \rightarrow \operatorname{Aut}(\mathfrak{g})$ is the adjoint representation of $G$ on $\mathfrak{g}$, let $q$ be an $\operatorname{Ad}_{G^{-}}$ invariant scalar product on $\mathfrak{g}$, and $\mathfrak{m}=\mathfrak{k}^{\perp}$ be the orthogonal complement to $\mathfrak{k}$ with respect to $q$. Then $\mathfrak{g}=\mathfrak{k} \oplus \mathfrak{m}$ is a reductive decomposition of $\mathfrak{g}$, that is, $[\mathfrak{k}, \mathfrak{m}] \subset \mathfrak{m}$. We also identify $\mathfrak{m}$ with the tangent space $T_{o}(G / K)$ at the point $o=e K$. Then the isotropy representation of $K$ is identified with the restriction $\left.\operatorname{Ad}_{K}\right|_{\mathfrak{m}}$ of the adjoint representation of $K$ on $\mathfrak{g}$ to $\mathfrak{m}$.

A $G$-invariant metric $g$ on $M=G / K$ corresponds to an $\operatorname{Ad}_{K}$-invariant scalar product $\langle$,$\rangle on \mathfrak{m}=T_{o} M$, and conversely any $\operatorname{Ad}_{K}$-invariant scalar product $\langle$,$\rangle on$ $\mathfrak{m}$ determines a $G$-invariant metric $g$ on $M=G / K$. The metric $g$ is called normal if the scalar product $\langle$,$\rangle on \mathfrak{m}$ is the restriction of an $\operatorname{Ad}_{G}$-invariant non-degenerate symmetric bilinear form $($,$) on \mathfrak{g}$. If $()=,-B(B=$ the Killing form on $\mathfrak{g})$, then the metric $g$ is called standard. Any $\operatorname{Ad}_{K}$-invariant scalar product $\langle$,$\rangle on \mathfrak{m}$ can be written as $\langle x, y\rangle=(A x, y)(x, y \in \mathfrak{m})$, where $A$ is an $\operatorname{Ad}_{K}$-invariant positive definite symmetric operator on $\mathfrak{m}$. Conversely, any such operator $A$ determines an $\operatorname{Ad}_{K}$-invariant scalar product $\langle\cdot, \cdot\rangle=(A \cdot, \cdot)$ on $\mathfrak{m}$, which defines a $G$-invariant Riemannian metric $g$ on $M$. We will say that $A$ is the operator associated with the metric $g$, or simply the associated operator. If $G$ is simple, then any normal metric on $G / K$ is proportional to the standard metric; hence it corresponds to the scalar operator $A=c \operatorname{Id}_{\mathfrak{m}}(c>0)$.

A Riemannian homogeneous manifold $(M=G / K, g)$ is called naturally reductive if there exists a reductive decomposition $\mathfrak{g}=\mathfrak{k} \oplus \mathfrak{m}$ such that for any $X \in \mathfrak{m}$ the operator $A_{X} \in \operatorname{End}(\mathfrak{m})$ defined by $A_{X}(Y)=[X, Y]_{\mathfrak{m}}$ is skew-symmetric with respect to $\langle$,$\rangle (here Z_{\mathfrak{m}}$ is the $\mathfrak{m}$-component of a vector $Z \in \mathfrak{g}=\mathfrak{k} \oplus \mathfrak{m}$ ). In this case the metric $g$ is called $G$-naturally reductive. It is well known that natural reductivity is equivalent to the geometrical property that for each vector $u \in \mathfrak{m}$, the orbit $\gamma(t)=(\exp t u)_{o}$ is a geodesic. The metric $g$ of a Riemannian manifold $(M, g)$ is called naturally reductive if $M$ admits a transitive group of isometries $G$ such that $g$ is a $G$-naturally reductive $G$-invariant metric on $M=G / K$. Clearly a normal metric is naturally reductive.

A result of Kostant (see also [Be, p. 196], DA-Zi, p. 4]) states that $(M=G / K, g)$ is naturally reductive if and only if there exists a transitive subgroup $G^{\prime} \subset G$ with Lie algebra $\mathfrak{g}^{\prime}=\mathfrak{m}+[\mathfrak{m}, \mathfrak{m}]$ (an ideal in $\mathfrak{g}$ ), such that $\left(M=G^{\prime} / K^{\prime}, g\right.$ ) is normal (here $K^{\prime}=K \cap G^{\prime}$ ). Notice that if $G$ is a compact simple Lie group, then any $G$-naturally reductive metric on $M=G / K$ is proportional to the standard metric.

In the following proposition we give equivalent algebraic conditions so that an orbit $\gamma(t)=(\exp t u) o(u \in \mathfrak{g})$ through the point $o \in G / K$ is a geodesic. Conditions 
(3) and (4) were originally established by B. Kostant Kos1 and E. B. Vinberg Vin, and reformulated by $\mathrm{O}$. Kowalski and L. Vanhecke [Ko-Va]. Our contribution is condition (2), which will be of special importance in our work.

Proposition 1. Let $(M=G / K, g)$ be a homogeneous Riemannian manifold and $A$ be the associated operator. Let $a \in \mathfrak{k}$ and $x \in \mathfrak{m}$. Then the following are equivalent:

(1) the orbit $\gamma(t)=\exp t(a+x) \cdot o$ of the one-parameter subgroup $\exp t(a+x)$ through the point $o=e K$ is a geodesic of $M$;

(2) $[a+x, A x] \in \mathfrak{k}$;

(3) $\langle[a, x], y\rangle=\left\langle x,[x, y]_{\mathfrak{m}}\right\rangle$ for all $y \in \mathfrak{m}$;

(4) $\left\langle[a+x, y]_{\mathfrak{m}}, x\right\rangle=0$ for all $y \in \mathfrak{m}$.

Proof. We show that (1) is equivalent to (2). For each $u \in \mathfrak{g}$, let $L_{u} \in \operatorname{End}(\mathfrak{m})$ be the Nomizu operator defined by

$$
2\left\langle L_{u} x, y\right\rangle=\left\langle[u, x]_{\mathfrak{m}}, y\right\rangle-\left\langle u_{m},[x, y]_{\mathfrak{m}}\right\rangle-\left\langle x,[u, y]_{\mathfrak{m}}\right\rangle
$$

for all $x, y \in \mathfrak{m}$. If $u \in \mathfrak{g}$, then Kostant and Vinberg have shown that $L_{u} u_{\mathfrak{m}}=$ $-\nabla_{u_{\mathrm{m}}} u^{*}$, where $u^{*}$ is the fundamental vector field associated to $u$, i.e., $u^{*}=$ $\left.\frac{d}{d t}(\exp t u \cdot o)\right|_{t=0}$. Hence if $u=a+x(a \in \mathfrak{k}, x \in \mathfrak{m})$, then the orbit $\gamma(t)=(\exp t u) o$ is a geodesic through $o$ if and only if

$$
\left.\nabla_{\dot{\gamma}(0)} \dot{\gamma}(t)\right|_{t=0}=-L_{u} u_{\mathfrak{m}}=L_{a+x} x=0 .
$$

Equation (2) reduces to

$$
\begin{aligned}
0 & =\langle[a, x], y\rangle-\left\langle x,[x, y]_{\mathfrak{m}}\right\rangle=B(A[a, x], y)-B\left(A x,[x, y]_{\mathfrak{m}}\right) \\
& =B\left(A\left(\operatorname{ad}_{a} x\right), y\right)-B\left(A x, \operatorname{ad}_{x} y\right)=B\left(A\left(\operatorname{ad}_{a} x, y\right)\right)+B\left(\operatorname{ad}_{x}(A x), y\right)
\end{aligned}
$$

for all $y \in \mathfrak{m}$. By the $\operatorname{Ad}_{K}$-invariance of the operator $A$ (i.e., $\left[\operatorname{ad}_{a}, A\right]=0$ for all $a \in \mathfrak{k})$ the last equality is equivalent to $\operatorname{ad}_{a} A x+\operatorname{ad}_{x} A x \in \mathfrak{k}$, or $[a+x, A x] \in \mathfrak{k}$, which completes the proof. The equivalence of (1) and (3) was established through the proof of $(1) \Leftrightarrow(2)$. Finally, the equivalence of (3) and (4) is obtained by the the following computation:

$$
\begin{aligned}
0 & =\left\langle[a+x, y]_{\mathfrak{m}}, x\right\rangle=\left\langle[a, y]_{\mathfrak{m}}, x\right\rangle+\left\langle[x, y]_{\mathfrak{m}}, x\right\rangle \\
& =-\langle[y, a], x\rangle+\left\langle[x, y]_{\mathfrak{m}}, x\right\rangle=-\langle y,[a, x]\rangle+\left\langle[x, y]_{\mathfrak{m}}, x\right\rangle .
\end{aligned}
$$

Definition. A Riemannian homogeneous manifold $(M=G / K, g)$ has homogeneous geodesics if each geodesic of $M$ is homogeneous, i.e., an orbit of a oneparameter group of isometries $\{(\exp t X) o: X \in \mathfrak{g}\}$.

An immediate important corollary of Proposition 1 is the following.

Corollary 2. Let $(M=G / K, g)$ be a homogeneous Riemannian manifold. Then $M$ has homogeneous geodesics if and only if for every $x \in \mathfrak{m}$ there exists an $a(x) \in \mathfrak{k}$ such that

$$
[a(x)+x, A x] \in \mathfrak{k} .
$$




\section{FLAG MANIFOLDS}

A description of flag manifolds in terms of painted Dynkin diagrams. A homogeneous manifold $M=G / K$ of a compact semisimple Lie group $G$ is called a flag manifold if it is isomorphic to an adjoint orbit of the group $G$. Equivalently, the isotropy subgroup $K$ is the centralizer of a torus in $G$.

Next we give the Lie-theoretic description of flag manifolds in terms of their painted Dynkin diagrams (see also $[\mathrm{A}-\mathrm{P}, \mathrm{A}, \mathrm{B}-\mathrm{F}-\mathrm{R}]$, Gor-On-Vin]).

We assume that $G$ is simple without center with Lie algebra $\mathfrak{g}$. Let

$$
\mathfrak{g}^{\mathbb{C}}=\mathfrak{h}^{\mathbb{C}} \oplus \sum_{\alpha \in R} \mathbb{C} E_{\alpha}
$$

be the root space decomposition of the complexification $\mathfrak{g}^{\mathbb{C}}$ of $\mathfrak{g}$ with respect to a Cartan subalgebra $\mathfrak{h}^{\mathbb{C}}$ of $\mathfrak{g}^{\mathbb{C}}$. Here $R \subset\left(\mathfrak{h}^{\mathbb{C}}\right)^{*}$ is the root system of $\mathfrak{g}^{\mathbb{C}}$, and $E_{\alpha}$ are the root vectors. We identify the dual space $\left(\mathfrak{h}^{\mathbb{C}}\right)^{*}$ with $\mathfrak{h}^{\mathbb{C}}$ via the Killing form $B$ of $\mathfrak{g}^{\mathbb{C}}$.

Let $\Pi=\left\{\alpha_{1}, \ldots, \alpha_{l}\right\}$ be a system of simple roots of $R$. For any subsystem $\Pi_{K} \subset \Pi$ we denote by

$$
R_{K}=\left\{\beta \in R: \beta=\sum_{\alpha_{i} \in \Pi_{K}} k_{i} \alpha_{i}\right\}
$$

the closed subsystem of roots spanned by $\Pi_{K}$, and by

$$
\mathfrak{k}^{\mathbb{C}}\left(\Pi_{K}\right)=\mathfrak{k}^{\mathbb{C}}=\mathfrak{h}^{\mathbb{C}} \oplus \sum_{\beta \in R_{K}} \mathbb{C} E_{\beta}
$$

the associated reductive subalgebra of $\mathfrak{g}^{\mathbb{C}}$. Let $K$ be the connected subgroup of $G$ generated by the subalgebra $\mathfrak{k}=\mathfrak{k}^{\mathbb{C}} \cap \mathfrak{g}$. Then the homogeneous manifold $M=$ $G / K$ is a flag manifold, and any flag manifold is of such a form. We denote by $R_{M}=R \backslash R_{K}$ the set complementary to the roots $R_{K}$. Then

$$
\mathfrak{g}^{\mathbb{C}}=\mathfrak{k}^{\mathbb{C}}+\mathfrak{m}^{\mathbb{C}} \text { where } \mathfrak{m}^{\mathbb{C}}=\sum_{\alpha \in R_{M}} \mathbb{C} E_{\alpha}
$$

is a reductive decomposition of $\mathfrak{g}^{\mathbb{C}}$. The root vectors $\left\{E_{\beta} \in \mathfrak{g}_{\alpha}: \beta \in R_{M}\right\}$ form a basis of the space $\mathfrak{m}^{\mathbb{C}}$, which is naturally identified with the complexified tangent space $\left(T_{o}^{M}\right)^{\mathbb{C}}$ of the flag manifold $M$. The reductive decomposition of the compact Lie algebra $\mathfrak{g}$ is given by

$$
\mathfrak{g}=\mathfrak{k}+\mathfrak{m} \text { where } \mathfrak{m}=\mathfrak{m}^{\mathbb{C}} \cap \mathfrak{g} .
$$

Let $\Gamma=\Gamma(\Pi)$ be the Dynkin diagram of the simple root system $\Pi$. By painting nodes of $\Gamma$ corresponding to $\Pi \backslash \Pi_{K}$ in black, we obtain the painted Dynkin diagram of $M=G / K$. In this diagram the system $\Pi_{K}$ is determined as the subdiagram of white roots.

Definition. Two $G$-manifolds $M=G / K$ and $M^{\prime}=G / K^{\prime}$ are called equivalent if there exists an automorphism $\alpha \in \operatorname{Aut}(G)$ such that $\alpha(K)=K^{\prime}$.

Such an automorphism defines a diffeomorphism $\tilde{\alpha}: M \rightarrow M^{\prime}$ given by $\tilde{\alpha}(g K)=$ $\alpha(g) K^{\prime}$, which satisfies $\tilde{\alpha}(g x)=\alpha(g) \tilde{\alpha}(x)$ for all $g \in G, x \in M$. If $g^{\prime}$ is a $G$-invariant metric on $M^{\prime}$, then $g=\tilde{\alpha}^{*}\left(g^{\prime}\right)$ is a $G$-invariant metric on $M$, and $\tilde{\alpha}:(M, g) \rightarrow$ $\left(M, g^{\prime}\right)$ is an isometry. 
Proposition 3 ([A]). Different painted connected Dynkin diagrams $\Gamma$ and $\Gamma_{1}$ (except for the case of $D_{\ell}$ ) define equivalent flag manifolds $G / K$ and $G / K^{\prime}$ if the subdiagrams $\Gamma^{\prime}$ and $\Gamma_{1}^{\prime}$ of white roots corresponding to $\Pi_{K}$ and $\Pi_{K^{\prime}}$ are isomorphic.

By using this procedure it is possible to give a complete list of all flag manifolds $G / K$, where $G$ is either a classical or an exceptional Lie group (up to isomorphism).

Flag manifolds of a classical Lie group.

$$
\begin{gathered}
A(\bar{n})=S U(n) / S\left(U\left(n_{1}\right) \cdots U\left(n_{s}\right)\right), \\
\bar{n}=\left(n_{1}, \ldots, n_{s}\right), n=n_{1}+\cdots+n_{s}, n_{1} \geq n_{2} \geq \cdots \geq n_{s} \geq 1, \\
B(\bar{\ell})=S O(2 \ell+1) / U\left(\ell_{1}\right) \cdots U\left(\ell_{k}\right) \cdot S O(2 m+1), \\
C(\bar{\ell})=S p(\ell) / U\left(\ell_{1}\right) \cdots U\left(\ell_{k}\right) \cdot S p(m), \\
D(\bar{\ell})=S O(2 \ell) / U\left(\ell_{1}\right) \cdots U\left(\ell_{k}\right) \cdot S O(2 m), \\
\bar{\ell}=\left(\ell_{1}, \ldots, \ell_{k}, m\right), \ell=\ell_{1}+\cdots+\ell_{k}+m, \ell_{1} \geq \cdots \geq \ell_{k} \geq 1, k, m \geq 0 .
\end{gathered}
$$

Flag manifolds of an exceptional Lie group. The subsequent list of flag manifolds $G / K$ determined by an exceptional Lie group $G$ consists of 101 non-isomorphic spaces and is to be read as follows. Let $\Gamma(\Pi)$ be the Dynkin diagram of the simple root system $\Pi=\left\{\alpha_{1}, \ldots, \alpha_{l}\right\}$. Then $G\left(\alpha_{1}, \ldots, \alpha_{k}\right)$ means that $\Pi_{K}=\left\{\alpha_{1}, \ldots, \alpha_{k}\right\}$, which corresponds to the semisimple part $\mathfrak{k}^{\prime}$ of $\mathfrak{k}$, and the remaining $l-k$ nodes of $\Gamma(\Pi)$ have been painted black, so that

$$
\mathfrak{k}=\mathfrak{u}(1) \oplus \cdots \oplus \mathfrak{u}(1) \oplus \mathfrak{k},
$$

with $l-k$ copies of $\mathfrak{u}(1)$. The case where all nodes of $\Gamma(\Pi)$ have been painted black corresponds to the manifold $G / T$ of full flag, where $T$ is a maximal torus in $G$.

For example, $E_{6}\left(\alpha_{2}, \alpha_{3}, \alpha_{4}, \alpha_{6}\right)$ corresponds to the painted Dynkin diagram

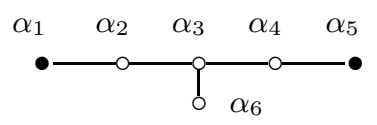

or to the flag manifold $E_{6} / S O(8) \cdot U(1) \cdot U(1)$.

The list of flag manifolds determined by an exceptional Lie group $G$ is the following:

$$
\begin{aligned}
& G_{2}(0), G_{2}\left(\alpha_{1}\right), G_{2}\left(\alpha_{2}\right) \\
& F_{4}(0), \quad F_{4}\left(\alpha_{4}\right), F_{4}\left(\alpha_{1}\right), F_{4}\left(\alpha_{3}, \alpha_{4}\right), F_{4}\left(\alpha_{1}, \alpha_{2}\right), F_{4}\left(\alpha_{1}, \alpha_{4}\right), F_{4}\left(\alpha_{2}, \alpha_{3}\right), \\
& F_{4}\left(\alpha_{1}, \alpha_{3}, \alpha_{4}\right), F_{4}\left(\alpha_{1}, \alpha_{2}, \alpha_{4}\right), F_{4}\left(\alpha_{2}, \alpha_{3}, \alpha_{4}\right), F_{4}\left(\alpha_{1}, \alpha_{2}, \alpha_{3}\right) \\
& E_{6}(0), E_{6}\left(\alpha_{5}\right), E_{6}\left(\alpha_{4}, \alpha_{5}\right), E_{6}\left(\alpha_{3}, \alpha_{5}\right), E_{6}\left(\alpha_{3}, \alpha_{4}, \alpha_{5}\right), E_{6}\left(\alpha_{2}, \alpha_{4}, \alpha_{5}\right), \\
& E_{6}\left(\alpha_{1}, \alpha_{3}, \alpha_{5}\right), E_{6}\left(\alpha_{2}, \alpha_{3}, \alpha_{4}, \alpha_{5}\right), E_{6}\left(\alpha_{1}, \alpha_{3}, \alpha_{4}, \alpha_{5}\right), E_{6}\left(\alpha_{1}, \alpha_{2}, \alpha_{4}, \alpha_{5}\right), \\
& E_{6}\left(\alpha_{2}, \alpha_{4}, \alpha_{5}, \alpha_{6}\right), E_{6}\left(\alpha_{2}, \alpha_{3}, \alpha_{4}, \alpha_{6}\right), E_{6}\left(\alpha_{1}, \alpha_{2}, \alpha_{3}, \alpha_{4}, \alpha_{5}\right), \\
& E_{6}\left(\alpha_{1}, \alpha_{2}, \alpha_{3}, \alpha_{4}, \alpha_{6}\right), E_{6}\left(\alpha_{1}, \alpha_{3}, \alpha_{4}, \alpha_{5}, \alpha_{6}\right), E_{6}\left(\alpha_{1}, \alpha_{2}, \alpha_{4}, \alpha_{5}, \alpha_{6}\right)
\end{aligned}
$$


$E_{7}(0), E_{7}\left(\alpha_{6}\right), E_{7}\left(\alpha_{5}, \alpha_{6}\right), E_{7}\left(\alpha_{4}, \alpha_{6}\right), E_{7}\left(\alpha_{4}, \alpha_{5}, \alpha_{6}\right), E_{7}\left(\alpha_{3}, \alpha_{5}, \alpha_{6}\right)$,

$E_{7}\left(\alpha_{2}, \alpha_{4}, \alpha_{6}\right), E_{7}\left(\alpha_{4}, \alpha_{6}, \alpha_{7}\right), E_{7}\left(\alpha_{3}, \alpha_{4}, \alpha_{5}, \alpha_{6}\right), E_{7}\left(\alpha_{2}, \alpha_{4}, \alpha_{5}, \alpha_{6}\right)$,

$E_{7}\left(\alpha_{4}, \alpha_{5}, \alpha_{6}, \alpha_{7}\right), E_{7}\left(\alpha_{2}, \alpha_{3}, \alpha_{5}, \alpha_{6}\right), E_{7}\left(\alpha_{1}, \alpha_{3}, \alpha_{5}, \alpha_{6}\right), E_{7}\left(\alpha_{2}, \alpha_{4}, \alpha_{6}, \alpha_{7}\right)$,

$E_{7}\left(\alpha_{2}, \alpha_{3}, \alpha_{4}, \alpha_{7}\right), E_{7}\left(\alpha_{2}, \alpha_{3}, \alpha_{4}, \alpha_{5}, \alpha_{6}\right), E_{7}\left(\alpha_{3}, \alpha_{4}, \alpha_{5}, \alpha_{6}, \alpha_{7}\right)$,

$E_{7}\left(\alpha_{1}, \alpha_{3}, \alpha_{4}, \alpha_{5}, \alpha_{6}\right), E_{7}\left(\alpha_{1}, \alpha_{2}, \alpha_{4}, \alpha_{5}, \alpha_{6}\right), E_{7}\left(\alpha_{2}, \alpha_{4}, \alpha_{5}, \alpha_{6}, \alpha_{7}\right)$,

$E_{7}\left(\alpha_{2}, \alpha_{3}, \alpha_{4}, \alpha_{6}, \alpha_{7}\right), E_{7}\left(\alpha_{1}, \alpha_{2}, \alpha_{5}, \alpha_{6}, \alpha_{7}\right)$,

$E_{7}\left(\alpha_{1}, \alpha_{2}, \alpha_{4}, \alpha_{6}, \alpha_{7}\right), E_{7}\left(\alpha_{1}, \alpha_{2}, \alpha_{3}, \alpha_{4}, \alpha_{7}\right), E_{7}\left(\alpha_{1}, \alpha_{2}, \alpha_{3}, \alpha_{4}, \alpha_{5}, \alpha_{6}\right)$,

$E_{7}\left(\alpha_{2}, \alpha_{3}, \alpha_{4}, \alpha_{5}, \alpha_{6}, \alpha_{7}\right), E_{7}\left(\alpha_{1}, \alpha_{3}, \alpha_{4}, \alpha_{5}, \alpha_{6}, \alpha_{7}\right), E_{7}\left(\alpha_{1}, \alpha_{2}, \alpha_{4}, \alpha_{5}, \alpha_{6}, \alpha_{7}\right)$,

$E_{7}\left(\alpha_{1}, \alpha_{2}, \alpha_{3}, \alpha_{5}, \alpha_{6}, \alpha_{7}\right), E_{7}\left(\alpha_{1}, \alpha_{2}, \alpha_{3}, \alpha_{4}, \alpha_{6}, \alpha_{7}\right), E_{7}\left(\alpha_{1}, \alpha_{2}, \alpha_{3}, \alpha_{4}, \alpha_{5}, \alpha_{7}\right)$

$E_{8}(0), E_{8}\left(\alpha_{7}\right), E_{8}\left(\alpha_{6}, \alpha_{7}\right), E_{8}\left(\alpha_{5}, \alpha_{7}\right), E_{8}\left(\alpha_{5}, \alpha_{6}, \alpha_{7}\right), E_{8}\left(\alpha_{4}, \alpha_{6}, \alpha_{7}\right)$,

$E_{8}\left(\alpha_{3}, \alpha_{5}, \alpha_{7}\right), E_{8}\left(\alpha_{4}, \alpha_{5}, \alpha_{6}, \alpha_{7}\right), E_{8}\left(\alpha_{3}, \alpha_{5}, \alpha_{6}, \alpha_{7}\right), E_{8}\left(\alpha_{3}, \alpha_{4}, \alpha_{6}, \alpha_{7}\right)$,

$E_{8}\left(\alpha_{2}, \alpha_{4}, \alpha_{6}, \alpha_{7}\right), E_{8}\left(\alpha_{1}, \alpha_{3}, \alpha_{5}, \alpha_{7}\right), E_{8}\left(\alpha_{2}, \alpha_{3}, \alpha_{4}, \alpha_{8}\right), E_{8}\left(\alpha_{3}, \alpha_{4}, \alpha_{5}, \alpha_{6}, \alpha_{7}\right)$,

$E_{8}\left(\alpha_{2}, \alpha_{4}, \alpha_{5}, \alpha_{6}, \alpha_{7}\right), E_{8}\left(\alpha_{2}, \alpha_{3}, \alpha_{5}, \alpha_{6}, \alpha_{7}\right), E_{8}\left(\alpha_{1}, \alpha_{3}, \alpha_{5}, \alpha_{6}, \alpha_{7}\right)$,

$E_{8}\left(\alpha_{1}, \alpha_{3}, \alpha_{4}, \alpha_{6}, \alpha_{7}\right), E_{8}\left(\alpha_{2}, \alpha_{4}, \alpha_{6}, \alpha_{7}, \alpha_{8}\right), E_{8}\left(\alpha_{2}, \alpha_{3}, \alpha_{4}, \alpha_{7}, \alpha_{8}\right)$,

$E_{8}\left(\alpha_{1}, \alpha_{2}, \alpha_{3}, \alpha_{4}, \alpha_{8}\right), E_{8}\left(\alpha_{2}, \alpha_{3}, \alpha_{4}, \alpha_{5}, \alpha_{6}, \alpha_{7}\right), E_{8}\left(\alpha_{1}, \alpha_{3}, \alpha_{4}, \alpha_{5}, \alpha_{6}, \alpha_{7}\right)$,

$E_{8}\left(\alpha_{1}, \alpha_{2}, \alpha_{4}, \alpha_{5}, \alpha_{6}, \alpha_{7}\right), E_{8}\left(\alpha_{1}, \alpha_{2}, \alpha_{3}, \alpha_{5}, \alpha_{6}, \alpha_{7}\right), E_{8}\left(\alpha_{2}, \alpha_{4}, \alpha_{5}, \alpha_{6}, \alpha_{7}, \alpha_{8}\right)$,

$E_{8}\left(\alpha_{2}, \alpha_{3}, \alpha_{4}, \alpha_{6}, \alpha_{7}, \alpha_{8}\right), E_{8}\left(\alpha_{1}, \alpha_{2}, \alpha_{3}, \alpha_{4}, \alpha_{7}, \alpha_{8}\right), E_{8}\left(\alpha_{2}, \alpha_{3}, \alpha_{4}, \alpha_{5}, \alpha_{6}, \alpha_{8}\right)$,

$E_{8}\left(\alpha_{1}, \alpha_{2}, \alpha_{5}, \alpha_{6}, \alpha_{7}, \alpha_{8}\right), E_{8}\left(\alpha_{1}, \alpha_{2}, \alpha_{4}, \alpha_{6}, \alpha_{7}, \alpha_{8}\right), E_{8}\left(\alpha_{1}, \alpha_{2}, \alpha_{3}, \alpha_{4}, \alpha_{5}, \alpha_{8}\right)$,

$E_{8}\left(\alpha_{1}, \alpha_{2}, \alpha_{3}, \alpha_{4}, \alpha_{5}, \alpha_{6}, \alpha_{7}\right), E_{8}\left(\alpha_{2}, \alpha_{3}, \alpha_{4}, \alpha_{5}, \alpha_{6}, \alpha_{7}, \alpha_{8}\right)$,

$E_{8}\left(\alpha_{1}, \alpha_{3}, \alpha_{4}, \alpha_{5}, \alpha_{6}, \alpha_{7}, \alpha_{8}\right), E_{8}\left(\alpha_{1}, \alpha_{2}, \alpha_{4}, \alpha_{5}, \alpha_{6}, \alpha_{7}, \alpha_{8}\right)$,

$E_{8}\left(\alpha_{1}, \alpha_{2}, \alpha_{3}, \alpha_{5}, \alpha_{6}, \alpha_{7}, \alpha_{8}\right), E_{8}\left(\alpha_{1}, \alpha_{2}, \alpha_{3}, \alpha_{4}, \alpha_{6}, \alpha_{7}, \alpha_{8}\right)$,

$E_{8}\left(\alpha_{1}, \alpha_{2}, \alpha_{3}, \alpha_{4}, \alpha_{5}, \alpha_{7}, \alpha_{8}\right), E_{8}\left(\alpha_{1}, \alpha_{2}, \alpha_{3}, \alpha_{4}, \alpha_{5}, \alpha_{6}, \alpha_{8}\right)$.

$T$-roots, isotropy representation and $G$-invariant metrics. Let $M=G / K$ be a flag manifold, and $\mathfrak{g}, \mathfrak{k}$ be the Lie algebras of the groups $G$ and $K$ respectively. Let $\mathfrak{h}^{\mathbb{C}}$ be a Cartan subalgebra of the complexification $\mathfrak{k}^{\mathbb{C}}$ of $\mathfrak{k}$, which is also a Cartan subalgebra of $\mathfrak{g}^{\mathbb{C}}$. Then we have the following Cartan decompositions:

$$
\mathfrak{g}^{\mathbb{C}}=\mathfrak{h}^{\mathbb{C}} \oplus \sum_{\alpha \in R} \mathfrak{g}_{\alpha}, \quad \mathfrak{k}^{\mathbb{C}}=\mathfrak{h}^{\mathbb{C}} \oplus \sum_{\alpha \in R_{K}} \mathfrak{g}_{\alpha}, \quad \mathfrak{m}^{\mathbb{C}}=\sum_{\alpha \in R_{M}} \mathfrak{g}_{\alpha},
$$

where $R$ (respectively $R_{K}$ ) is the root system of $\mathfrak{g}^{\mathbb{C}}$ (respectively of $\mathfrak{k}^{\mathbb{C}}$ ) with respect to $\mathfrak{h}^{\mathbb{C}}$.

We denote by $\mathfrak{h}=\mathfrak{h}^{\mathbb{C}} \cap i \mathfrak{k}$ the real ad-diagonal subalgebra, and by

$$
\mathfrak{t}=Z\left(\mathfrak{k}^{\mathbb{C}}\right) \cap i \mathfrak{h}
$$

the intersection of the center $Z\left(\mathfrak{k}^{\mathbb{C}}\right)$ with $i \mathfrak{h}$. Then $\mathfrak{k}^{\mathbb{C}}=\mathfrak{t}^{\mathbb{C}} \oplus \mathfrak{k}^{\mathbb{C}}$, where $\mathfrak{k}^{\prime \mathbb{C}}$ is the semisimple part of $\mathfrak{k}^{\mathbb{C}}$.

We consider the restriction map

$$
\kappa: \mathfrak{h}^{*} \rightarrow \mathfrak{t}^{*},\left.\quad \alpha \mapsto \alpha\right|_{\mathfrak{t}}
$$

and set $R_{T}=\kappa\left(R_{M}\right)$. 
Definition. The elements of $R_{T}$ are called T-roots.

It is known ( $[\mathrm{Sie},[\mathrm{A}-\mathrm{P}]$ ) that there exists a 1-1 correspondence between $T$-roots $\xi$ and irreducible submodules $\mathfrak{m}_{\xi}$ of the $\operatorname{Ad}_{K}$-module $\mathfrak{m}^{\mathbb{C}}$, given by

$$
R_{T} \ni \xi \leftrightarrow \mathfrak{m}_{\xi}=\sum_{\kappa(\alpha)=\xi} \mathfrak{g}_{\alpha} .
$$

Hence, a decomposition of the $\operatorname{Ad}_{K}$-module $\mathfrak{m}^{\mathbb{C}}$ into irreducible submodules is given by

$$
\mathfrak{m}^{\mathbb{C}}=\sum_{\xi \in R_{T}} \mathfrak{m}_{\xi} .
$$

We remark that the complex conjugation $\tau$ of $\mathfrak{g}^{\mathbb{C}}$ with respect to $\mathfrak{g}$ interchanges $\mathfrak{g}_{\alpha}$ and $\mathfrak{g}_{-\alpha}$, hence also $\mathfrak{m}_{\xi}$ and $\mathfrak{m}_{-\xi}$. Due to this, a decomposition of the real $\operatorname{Ad}_{K}$-module $\mathfrak{m}=\left(\mathfrak{m}^{\mathbb{C}}\right)^{\tau}$ into irreducible submodules is given by

$$
\mathfrak{m}=\sum_{\xi \in R_{T}^{+}}\left(\mathfrak{m}_{\xi}+\mathfrak{m}_{-\xi}\right)^{\tau} .
$$

Here $R_{T}^{+}=\kappa\left(R^{+}\right)$is the set of all positive $T$-roots (i.e., the restriction of the system $R^{+}$of positive roots to $\left.\mathfrak{t}\right), \tau$ is the complex conjugation of $\mathfrak{g}^{\mathbb{C}}$ with respect to $\mathfrak{g}$, and $\mathfrak{n}^{\tau}$ denotes the set of fixed points of $\tau$ in a vector subspace $\mathfrak{n} \subset \mathfrak{g}^{\mathbb{C}}$. An explicit description of the set of $T$-roots is obtained as follows: we fix a basis $\Pi=\left\{\alpha_{1}, \ldots, \alpha_{m}, \phi_{1}, \ldots, \phi_{k}\right\}$ of the root system $R$ such that $\Pi_{K}=\left\{\phi_{1}, \ldots \phi_{k}\right\}$ is a basis of the root system $R_{K}$, and we denote by $\pi_{1}, \pi_{2}, \ldots, \pi_{m}$ the fundamental weights associated to the simple roots $\alpha_{1}, \alpha_{2}, \ldots, \alpha_{m}$, i.e., linear forms defined by

$$
\frac{2\left(\pi_{j}, \alpha_{i}\right)}{\left(\alpha_{i}, \alpha_{i}\right)}=\delta_{i j}, \quad\left(\pi_{j}, \phi_{i}\right)=0 .
$$

Here $(\cdot, \cdot)$ is the restriction of the Killing form to $\mathfrak{h}^{*}$.

The fundamental weights $\pi_{i}(i=1, \ldots, m)$ form a basis of the space $\mathfrak{t}^{*}$ (isomorphic to $\mathfrak{t}$ via the Killing form). So the $T$-root $\bar{\alpha}=\kappa(\alpha)=\left.\alpha\right|_{\mathfrak{t}}$ associated to a root $\alpha \in R_{M}$, is given by

$$
\kappa(\alpha)=\sum_{j=1}^{m} \frac{2\left(\alpha, \alpha_{j}\right)}{\left(\alpha_{j}, \alpha_{j}\right)} \pi_{j} .
$$

Now following M.M. Graev, we prove the following lemma about the lift of relations between $T$-roots to $R$, which will be used in the next section.

Lemma 4 (M. M. Graev, unpublished). Let $\xi, \eta, \zeta$ be $T$-roots such that $\xi+\eta+\zeta=0$. Then there exist roots $\alpha, \beta, \gamma \in R$ with $\kappa(\alpha)=\xi, \kappa(\beta)=\eta, \kappa(\gamma)=\zeta$, and such that $\alpha+\beta+\gamma=0$.

Proof. Let $\Pi=\left\{\alpha_{1}, \ldots, \alpha_{m}, \phi_{1}, \ldots, \phi_{k}\right\}$ be a basis of the root system $R$ with $\alpha_{i} \in R_{M}(i=1, \ldots, m)$ and $\phi_{i} \in \Pi_{K}(i=1, \ldots, k)$. We denote by $\alpha \in$ $\kappa^{-1}(\xi), \beta \in \kappa^{-1}(\eta), \gamma \in \kappa^{-1}(\zeta)$ the roots which are the lowest weights of the $\mathfrak{k}^{s}$ modules $\mathfrak{m}_{\xi}, \mathfrak{m}_{\eta}, \mathfrak{m}_{\zeta}$ respectively, where $\mathfrak{k}^{s}$ is the semisimple part of the reductive Lie algebra $\mathfrak{k}^{\mathbb{C}}$. Without loss of generality we may assume that the roots $\alpha, \beta$ are positive and $\gamma$ is negative. Since $\kappa(\alpha+\beta+\gamma)=0$, we can write

$$
\alpha+\beta+\gamma=-\sum k_{i} \phi_{i},
$$


where the coefficients $k_{i}$ are integers. Moreover, $k_{i} \geq 0$ since $-(\alpha+\beta+\gamma)$ is a dominant weight of $\mathfrak{k}^{s}$. We can represent the positive root $-\gamma$ as a sum of positive roots as

$$
-\gamma=\alpha+\beta+\sum k_{i} \phi_{i}
$$

It is known Bour, Ch. VI, paragraph 1.6] that we can rearrange the summand in this formula in such a way so that, for any $n=1,2,3, \ldots$, all successive summands from the first term until the $n$-th term are roots. If

$$
-\gamma=\sum p_{i} \phi_{i}+\alpha+\sum q_{j} \phi_{j}+\beta+\sum r_{s} \phi_{s}
$$

is such a reordering, we put

$$
\alpha^{\prime}=\sum p_{i} \phi_{i}+\alpha+\sum q_{j} \phi_{j}, \beta^{\prime}=\beta, \gamma^{\prime}=\gamma+\sum r_{s} \phi_{s}
$$

Then $\alpha^{\prime}, \beta^{\prime}, \gamma^{\prime}$ is the desired triple of roots.

\section{Homogeneous geodesics in Riemannian Flag manifolds: \\ A NECESSARY CONDITION}

Recall that a $G$-invariant Riemannian metric $g$ on a homogeneous space $M=$ $G / K$ of a compact group $G$ with reductive decomposition $\mathfrak{g}=\mathfrak{k}+\mathfrak{m}$ is determined by an $\operatorname{Ad}_{K}$-invariant scalar product $\langle\cdot, \cdot\rangle=(A \cdot, \cdot)$ on $\mathfrak{m}$, where $A$ is the associated operator. We extend $A$ to a complex linear operator $A$ on $\mathfrak{m}^{\mathbb{C}}$.

Definition. A flag manifold $M=G / K$ equipped with a $G$-invariant metric $g$ is called a Riemannian flag manifold.

If $G / K$ is a Riemannian flag manifold, then by using decomposition (5) we can express $A$ as

$$
A=\left.\sum_{\xi \in R_{T}^{+}} \lambda_{\xi} \mathrm{Id}\right|_{\left(\mathfrak{m}_{\xi} \oplus \mathfrak{m}_{-\xi}\right)^{\tau}}
$$

We remark that $\lambda_{\xi}$ are the eigenvalues of the operator $A$ (positive constants), and that the scalar operator $A=c \operatorname{Id}_{\mathfrak{m}}$ corresponds to the standard metric on $M=G / K$.

Let $(M=G / K, g)$ be a Riemannian flag manifold with associated operator $A$ given by (6). We will give a necessary condition that all geodesics in $M$ through $o=e K$ are homogeneous geodesics, in terms of the set of $T$-roots.

Proposition 5. Let $(M=G / K, g)$ be a Riemannian flag manifold, A the associated operator given by (6), and let $\left\{\lambda_{\xi}: \xi \in R_{T}^{+}\right\}$be its eigenvalues. Assume that all geodesics of $(M, g)$ are homogeneous. Then if $\xi, \eta$ are two T-roots with $\eta$ different from $\pm \xi$ and $\pm 2 \xi$, and such that $\xi+\eta \in R_{T}$ or $\xi-\eta \in R_{T}$, then $\lambda_{\xi}=\lambda_{\eta}$.

Proof. By Corollary 2, for each $x \in \mathfrak{m}$ there exists an $a=a(x) \in \mathfrak{k}$ such that $[a+x, A x] \in \mathfrak{k}$. Assume that $\xi, \eta$ are such that $\xi+\eta \in R_{T}$. Similarly this works if $\xi-\eta \in R_{T}$. Let

$$
0 \neq x=x_{\xi}+x_{-\xi}+x_{\eta}+x_{-\eta} \in \mathfrak{m} \cap\left(\mathfrak{m}_{\xi}+\mathfrak{m}_{-\xi}+\mathfrak{m}_{\eta}+\mathfrak{m}_{-\eta}\right)
$$


such that $x_{\xi}, x_{\eta} \neq 0$. Since $\xi+\eta \in R_{T}$, then by Lemma $4,0 \neq\left[x_{\xi}, x_{\eta}\right] \in \mathfrak{m}_{\xi+\eta}$. Then condition (3) can be expressed as

$$
\begin{aligned}
{\left[a+x_{\xi}+x_{-\xi}+x_{\eta}+x_{-\eta}, \lambda_{\xi}\left(x_{\xi}+x_{-\xi}\right)+\lambda_{\eta}\left(x_{\eta}+x_{-\eta}\right)\right] } & \\
\equiv\left(\lambda_{\eta}-\lambda_{\xi}\right)\left(\left[x_{\xi}, x_{\eta}\right]+\left[x_{-\xi}, x_{-\eta}\right]\right. & +\left[x_{\xi}, x_{-\eta}+\left[x_{-\xi}, x_{\eta}\right]\right) \\
& \bmod \left(\mathfrak{m}_{\xi}+\mathfrak{m}_{\eta}+\mathfrak{m}_{-\xi}+\mathfrak{m}_{-\eta}+\mathfrak{k}\right) .
\end{aligned}
$$

Since the first term in the second bracket is a non-zero element of $\mathfrak{m}_{\xi+\eta}$, and the other terms belong to other $\operatorname{Ad}_{K}$-submodules, it follows that $\lambda_{\xi}=\lambda_{\eta}$.

Proposition 5 motivates the following:

Definition. (1) Two $T$-roots $\xi, \eta \in R_{T}$ are called adjacent if one of the following occurs:

(i) If $\eta$ is a multiple of $\xi$, then $\eta \neq 2 \xi$ and $\xi \neq 2 \eta$.

(ii) If $\eta$ is not a multiple of $\xi$, then either $\xi-\eta \in R_{T}$, or $\xi+\eta \in R_{T}$.

(2) Two $T$-roots $\xi, \eta$ are called connected if there exists a chain of $T$-roots

$$
\xi=\xi_{1}, \xi_{2}, \ldots, \xi_{s}=\eta
$$

such that $\xi_{i}, \xi_{i+1}$ are adjacent $(i=1, \ldots, s-1)$.

We remark that $\xi$ and $\pm \xi$ are connected, and if $\xi, 2 \xi$ are the only positive $T$-roots, then these are not connected. We define the relation

$$
\xi \sim \eta \Leftrightarrow \xi, \eta \text { are connected. }
$$

One can check easily that this is an equivalence relation. Let $R^{i}$ be the equivalence classes consisting of mutually connected $T$-roots. Then the set $R_{T}$ is decomposed into a disjoint union

$$
R_{T}=R^{1} \cup \cdots \cup R^{r} .
$$

Definition. The set of $T$-roots $R_{T}$ is called connected if $r=1$.

The above decomposition of $R_{T}$ induces the following decomposition for $\mathfrak{m}^{\mathbb{C}}$ :

$$
\mathfrak{m}^{\mathbb{C}}=\mathfrak{m}^{1} \oplus \cdots \oplus \mathfrak{m}^{r}
$$

where $\mathfrak{m}^{i}=\sum_{\xi \in R^{i}} \mathfrak{m}_{\xi}$. As a consequence we obtain the following:

Proposition 6. Let $(M=G / K, g)$ be a Riemannian flag manifold of a simple Lie group, and $\left\{\lambda_{\xi}: \xi \in R_{T}\right\}$ be the eigenvalues of the operator $A$ associated with the $G$-invariant metric $g$. If all geodesics of $(M, g)$ are homogeneous, then

$$
\lambda_{\xi}=\lambda_{\eta} \text { for all } \xi, \eta \in R^{i}(i=1, \ldots, r)
$$

In particular, the operator $A$ is expressed as

$$
A=\left.\lambda_{1} \operatorname{Id}\right|_{\mathfrak{m}^{1}}+\cdots+\left.\lambda_{r} \operatorname{Id}\right|_{\mathfrak{m}^{r}} .
$$

Proof. Let $R_{T}=R^{1} \cup \cdots \cup R^{r}$ be the decomposition of the set $R_{T}$, and let $\xi, \eta \in R^{i}$ for some $i=1, \ldots, r$. Then the result is immediate from Proposition 5 . Expression (7) for the operator $A$ is obtained from (5) and (6).

The above can be combined to the following important necessary condition:

Theorem 7. Let $M=G / K$ be a flag manifold of a simple Lie group. If the set of T-roots is connected, then the standard metric is the only (up to a scalar) $G$-invariant metric of $M$ with homogeneous geodesics. 


\section{Classification of flag manifolds of a Classical Lie group WITH NON-CONNECTED SYSTEM OF $T$-ROOTS}

Theorem 7 shows that if a flag manifold $M=G / K$ of a simple Lie group $G$ admits a $G$-invariant metric with homogeneous geodesics which is not homothetic to the standard metric, then the set $R_{T}$ of $T$-roots is not connected. In this section we describe all such manifolds of a classical Lie group $G$.

Let $G / K$ be a flag manifold, where $G$ is one of the classical Lie groups $A_{\ell}, B_{\ell}, C_{\ell}$ or $D_{\ell}$.

Case of $\mathbf{A}_{\ell}=S U(n), n=\ell+1$. A flag manifold of the group $A_{\ell}$ has the form

$$
A(\bar{n})=S U(n) / S\left(U\left(n_{1}\right) \cdots U\left(n_{s}\right)\right) .
$$

We describe the associated set of $T$-roots $R_{T}$ as follows (see $\left.\mathrm{A}-\mathrm{P}, \mathrm{A}\right]$ ).

Let $\epsilon=\left\{\epsilon_{1}, \ldots, \epsilon_{n}\right\}$ be the standard basis of $\mathbb{R}^{n}$. It is more convenient to pass to dual indices of the vectors of the basis $\epsilon$, so that $\epsilon=\left\{\epsilon_{1}^{1}, \ldots, \epsilon_{n_{1}}^{1}, \epsilon_{1}^{2}, \ldots, \epsilon_{n_{2}}^{2}, \ldots, \epsilon_{1}^{s}\right.$, $\left.\ldots, \epsilon_{n_{s}}^{s}\right\}$. Then the root system is given by

$$
R=\left\{\epsilon_{i}^{a}-\epsilon_{j}^{b}: i \neq j\right\}
$$

and if we take $R_{K}=\left\{\epsilon_{i}^{a}-\epsilon_{j}^{a}: i \neq j, 1 \leq a \leq s\right\}$, then

$$
R_{M}=\left\{\epsilon_{i}^{a}-\epsilon_{j}^{b}: a \neq b, i \neq j\right\}
$$

We also have that

$$
\begin{aligned}
& \mathfrak{t}=\left\{\operatorname{diag}\left(\epsilon^{1} \epsilon^{1} \cdots \epsilon^{1} \quad \epsilon^{2} \cdots \epsilon^{2} \cdots \epsilon^{s} \cdots e^{s}\right):\right. \\
& \left.\sum \epsilon^{i}=0, \epsilon^{i} \text { appears } n_{i} \text { times }(1 \leq i \leq s)\right\}
\end{aligned}
$$

Then the set of $T$-roots has the form

$$
R_{T}=\left\{\epsilon^{a}-\epsilon^{b}: a, b=1, \ldots, s\right\},
$$

which is a root system of type $A_{s-1}$. It is connected. Hence we get the following.

Proposition 8. The set of T-roots of the flag manifold $A(\bar{n})=S U(n) / S\left(U\left(n_{1}\right)\right.$ $\left.\cdots U\left(n_{s}\right)\right)$ is connected. Hence any $S U(n)$-invariant metric on $A(\bar{n})$ with homogeneous geodesics is homothetic to the standard metric.

Following $\mathrm{A}-\mathrm{P}$ we now describe the root systems $R, R_{K}$ for all flag manifolds $B(\bar{\ell}), C(\ell), D(\ell)$ of the classical groups $B_{\ell}=S O(2 \ell+1), C_{\ell}=S p(\ell)$, or $D_{\ell}=$ $S O(2 \ell)$ listed in Section 3.

Let $\epsilon=\left\{\epsilon_{i}^{a}, \pi_{j}\right\}$ be an orthonormal basis of $\mathbb{R}^{\ell}$, where $a=1, \ldots, k, j=1, \ldots, m$, and for a given $a$ the index $i$ takes the values $1, \ldots, \ell_{a}$. Then we can describe the root systems $R, R_{K}$ as follows:

$$
\begin{gathered}
R=\left\{ \pm \epsilon_{i}^{a} \pm \epsilon_{j}^{b}, \pm \epsilon_{i}^{a} \pm \epsilon_{j}^{a}, \pm \epsilon_{i}^{a} \pm \pi_{j}, \pm \pi_{i} \pm \pi_{j}, \pm \mu \epsilon_{i}^{a}, \pm \mu \pi_{j}: i<j, a<b\right\} \\
R_{K}=\left\{ \pm\left(\epsilon_{i}^{a}-\epsilon_{j}^{a}\right), \pm \pi_{i} \pm \pi_{j}, \pm \mu \pi_{j}\right\}
\end{gathered}
$$

where $\mu=1$ in the case of $B_{\ell}, \mu=2$ for $C_{\ell}$, and $\mu$ is absent for $D_{\ell}$.

Case of $\mathbf{B}_{\ell}$. We choose

$$
\begin{gathered}
R_{K}^{+}=\left\{\epsilon_{i}^{a}-\epsilon_{j}^{a}, \pi_{i} \pm \pi_{j}, \pi_{j}: i<j\right\} \text { so that } \\
R_{M}^{+}=R^{+} \backslash R_{K}^{+}=\left\{\epsilon_{i}^{a}+\epsilon_{j}^{a}, \epsilon_{i}^{a} \pm \epsilon_{j}^{b}, \epsilon_{i}^{a} \pm \pi_{j}, \epsilon_{i}^{a}: i<j, a<b\right\} .
\end{gathered}
$$


We note that $\mathfrak{t}=\left\{\operatorname{diag}\left(\epsilon^{1} \epsilon^{1} \cdots \epsilon^{1} \quad \epsilon^{2} \cdots \epsilon^{2} \cdots \epsilon^{k} \cdots e^{k}\right): \epsilon^{a}\right.$ appears $\ell_{a}$ times $(1 \leq a \leq k)\}$, so the set of positive $T$-roots is given by

$$
R_{T}^{+}=\left\{2 \epsilon^{a}, \epsilon^{a} \pm \epsilon^{b}, \epsilon^{a}: a<b, a, b=1, \ldots, k\right\} .
$$

If $\ell_{1}=\cdots=\ell_{k}=1$, then the vectors $2 \epsilon^{a}$ are absent, and $R_{T}^{+}$is connected. This corresponds to the space $S O(2 \ell+1) / U(1) \cdots U(1) \cdot S O(2 m+1)$. If $k=1$ and $\ell_{1} \neq 1$, it takes the form $R_{T}^{+}=\{2 \epsilon, \epsilon\}$, which is not connected. This corresponds to the space $B(\ell-m, m)=S O(2 \ell+1) / U(\ell-m) \cdot S O(2 m+1)$. In all other cases it is connected.

Case of $\mathbf{C}_{\ell}$. We choose

$$
\begin{gathered}
R_{K}^{+}=\left\{\epsilon_{i}^{a}-\epsilon_{j}^{a}, \pi_{i} \pm \pi_{j}, 2 \pi_{j}: i<j\right\} \text { so that } \\
R_{M}^{+}=\left\{\epsilon_{i}^{a}+\epsilon_{j}^{a}, \epsilon_{i}^{a} \pm \epsilon_{j}^{b}, \epsilon_{i}^{a} \pm \pi_{j}, 2 \epsilon_{i}^{a}: i<j, a<b\right\} .
\end{gathered}
$$

The set $\mathfrak{t}$ is the same as in $B_{\ell}$, so we obtain that

$$
R_{T}^{+}=\left\{2 \epsilon^{a}, \epsilon^{a} \pm \epsilon^{b}, \epsilon^{a}: a<b, a, b=1, \ldots, k\right\} .
$$

If $\ell_{1}=\cdots=\ell_{k}=1$, then the vectors $2 \epsilon^{a}$ are absent, and $R_{T}^{+}$is connected. This corresponds to the space $S p(2 \ell) / U(1) \cdots U(1) \cdot S p(m)$. If $k=1$ and $\ell_{1} \neq 1$, it takes the form $R_{T}^{+}=\{2 \epsilon, \epsilon\}$, which is not connected. This corresponds to the space $C(\ell-m, m)=S p(\ell) / U(\ell-m) \cdot S p(m)$. In all other cases it is connected.

Case of $\mathbf{D}_{\ell}$. We choose

$$
\begin{gathered}
R_{K}^{+}=\left\{\epsilon_{i}^{a}-\epsilon_{j}^{a}, \pi_{i} \pm \pi_{j}: i<j\right\}, \text { so that } \\
R_{M}^{+}=\left\{\epsilon_{i}^{a}+\epsilon_{j}^{a}, \epsilon_{i}^{a} \pm \epsilon_{j}^{b}, \epsilon_{i}^{a} \pm \pi_{j}: i<j, a<b\right\} .
\end{gathered}
$$

Similarly, it follows that

$$
R_{T}^{+}=\left\{2 \epsilon^{a}, \epsilon^{a} \pm \epsilon^{b}, \epsilon^{a}: a<b, a, b=1, \ldots, k\right\} .
$$

If $\ell_{1}=\cdots=\ell_{k}=1$, then as before the vectors $2 \epsilon^{a}$ are absent, and $R_{T}^{+}$is connected. This corresponds to the space $S O(2 \ell) / U(1) \cdots U(1) \cdot S O(2 m)$. If $k=$ 1 and $\ell_{1} \neq 1$, it takes the form $R_{T}^{+}=\{2 \epsilon, \epsilon\}$, which is not connected. This corresponds to the space $D(\ell-m, m)=S O(2 \ell) / U(\ell-m) \cdot S O(2 m)$. In all other cases it is connected. Hence we obtain:

Proposition 9. The only flag manifolds of the classical groups $B_{\ell}, C_{\ell}, D_{\ell}$ with non-connected system $R_{T}$ of T-roots are

$$
\begin{aligned}
& B(\ell-m, m)=S O(2 \ell+1) / U(\ell-m) \cdot S O(2 m+1), \\
& C(\ell-m, m)=S p(\ell) / U(\ell-m) \cdot S p(m), \\
& D(\ell-m, m)=S O(2 \ell) / U(\ell-m) \cdot S O(2 m) .
\end{aligned}
$$

For these manifolds $R_{T}=\{ \pm \epsilon, \pm 2 \epsilon\}$.

\section{Classification of flag manifolds of an exceptional Lie group WITH NON-CONNECTED SYSTEM OF T-ROOTS}

Now we determine all flag manifolds of an exceptional Lie group $G$ with nonconnected system $R_{T}$. Since there are many cases and the examinations are straightforward and elementary, we will present the case of the group $G_{2}$ in detail and consider only a few cases for the other groups $E_{4}, E_{6}, E_{7}, E_{8}$. We need the following description of the root systems $R$ of exceptional complex Lie algebras $\mathfrak{g}$, which we take from Gor-On-Vin. In the table is indicated the dimension of the exceptional 
Lie algebra, the set of simple roots $\Pi$, the maximal root $\mu$, and a description of the Weyl group $W$.

\begin{tabular}{|c|c|c|c|c|}
\hline Type & $\operatorname{dim} \mathfrak{g}$ & $R$ & $\Pi, \mu$ & $W$ \\
\hline$E_{8}$ & 248 & $\begin{array}{l}\varepsilon_{i}-\varepsilon_{j} \\
\pm\left(\varepsilon_{i}+\varepsilon_{j}+\varepsilon_{k}\right)\end{array}$ & $\begin{aligned} \alpha_{i} & =\varepsilon_{i}-\varepsilon_{i+1}, i<8 \\
\alpha_{8} & =\varepsilon_{6}+\varepsilon_{7}+\varepsilon_{8} \\
\mu & =\varepsilon_{1}-\varepsilon_{9}\end{aligned}$ & Aut $R$ \\
\hline$E_{7}$ & 133 & $\begin{array}{c}\varepsilon_{i}-\varepsilon_{j} \\
\varepsilon_{i}+\varepsilon_{j}+\varepsilon_{k}+\varepsilon_{l}\end{array}$ & $\begin{aligned} \alpha_{i} & =\varepsilon_{i}-\varepsilon_{i+1}, i<7 \\
\alpha_{7} & =\varepsilon_{5}+\varepsilon_{6}+\varepsilon_{7}+\varepsilon_{8} \\
\mu & =-\varepsilon_{7}+\varepsilon_{8}\end{aligned}$ & Aut $R$ \\
\hline$E_{6}$ & 78 & $\begin{array}{l}\varepsilon_{i}-\varepsilon_{j}, \pm 2 \varepsilon \\
\varepsilon_{i}+\varepsilon_{j}+\varepsilon_{k}+\varepsilon\end{array}$ & $\begin{aligned} \alpha_{i} & =\varepsilon_{i}-\varepsilon_{i+1}, i<6 \\
\alpha_{6} & =\varepsilon_{4}+\varepsilon_{5}+\varepsilon_{6}+\varepsilon \\
\mu & =2 \varepsilon\end{aligned}$ & Aut $R=W \times\{ \pm 1\}$ \\
\hline$F_{4}$ & 52 & $\begin{array}{l} \pm \varepsilon_{i} \pm \varepsilon_{j} ; \pm \varepsilon_{i} \\
\frac{1}{2}\left( \pm \varepsilon_{1} \pm \varepsilon_{2} \pm \varepsilon_{3} \pm \varepsilon_{4}\right)\end{array}$ & $\begin{aligned} \alpha_{1} & =\frac{1}{2}\left(\varepsilon_{1}-\varepsilon_{2}-\varepsilon_{3}-\varepsilon_{4}\right) \\
\alpha_{2} & =\varepsilon_{4} \\
\alpha_{3} & =\varepsilon_{3}-\varepsilon_{4} \\
\alpha_{4} & =\varepsilon_{2}-\varepsilon_{3} \\
\mu & =\varepsilon_{1}+\varepsilon_{2}\end{aligned}$ & Aut $R$ \\
\hline$G_{2}$ & 14 & $\varepsilon_{i}-\varepsilon_{j}, \pm \varepsilon_{i}$ & $\begin{aligned} \alpha_{1} & =-\varepsilon_{2} \\
\alpha_{2} & =\varepsilon_{2}-\varepsilon_{3} \\
\mu & =\varepsilon_{1}-\varepsilon_{3}\end{aligned}$ & Aut $R$ \\
\hline
\end{tabular}

The following notation is used. For $F_{4}, \quad \varepsilon_{i}(i=1,2,3,4)$ is an orthonormal basis of the 4-dimensional Euclidean space $\mathbb{R}^{4}$.

For all other exceptional root systems of rank $l, \varepsilon_{1}, \ldots, \varepsilon_{l+1}$ is the standard basis of the Euclidean vector space $\mathbb{R}^{l+1}$ restricted to the hyperplane

$$
E^{l}=\left\{\alpha=\sum x_{i} \varepsilon_{i}, \quad \sum_{i=1}^{l+1} x_{i}=0\right\} .
$$

In particular,

$$
\left(\varepsilon_{i}, \varepsilon_{i}\right)=\frac{l}{l+1}, \quad\left(\varepsilon_{i}, \varepsilon_{j}\right)=-\frac{1}{l+1}, \quad i \neq j .
$$

For $E_{6}, \varepsilon$ is the vector with $(\varepsilon, \varepsilon)=1 / 2$, orthogonal to all vectors $\varepsilon_{i}$.

Recall that every positive $T$-root is expressed as

$$
\operatorname{pr}_{\mathfrak{t}} \alpha:=k_{1} \bar{\alpha}_{1}+\cdots+k_{m} \bar{\alpha}_{m}
$$

where $\alpha \in R_{M}^{+}$, and $\bar{\alpha}_{i}=\operatorname{pr}_{\mathfrak{t}} \alpha_{i}=\left.\alpha_{i}\right|_{\mathfrak{t}}\left(\alpha_{i} \in \Pi \backslash \Pi_{K}\right)$.

Case of $\mathbf{G}_{\mathbf{2}}$. We fix an ordering so that the set of positive roots is

$$
R^{+}=\left\{-\left(2 \varepsilon_{2}+\varepsilon_{3}\right),-\left(\varepsilon_{2}+2 \varepsilon_{3}\right), \varepsilon_{2}-\varepsilon_{3},-\left(\varepsilon_{2}+\varepsilon_{3}\right),-\varepsilon_{2},-\varepsilon_{3}\right\} .
$$

The maximal root is $\mu=-\left(\varepsilon_{2}+2 \varepsilon_{3}\right)=3 \alpha_{1}+2 \alpha_{2}$, and any root $\alpha \in R$ is expressed as $\alpha=k_{1} \alpha_{1}+k_{2} \alpha_{2}$, with $\left|k_{1}\right| \leq 3$ and $\left|k_{2}\right| \leq 2$. More specifically we have:

$$
\begin{aligned}
& -\left(2 \varepsilon_{2}+\varepsilon_{3}\right)=3 \alpha_{1}+\alpha_{2},-\left(\varepsilon_{2}+2 \varepsilon_{3}\right)=3 \alpha_{1}+2 \alpha_{2}, \varepsilon_{2}-\varepsilon_{3}=\alpha_{2}, \\
& -\left(\varepsilon_{2}+\varepsilon_{3}\right)=2 \alpha_{1}+\alpha_{2},-\varepsilon_{2}=\alpha_{1},-\varepsilon_{3}=\alpha_{1}+\alpha_{2} .
\end{aligned}
$$

(1) $G_{2}(0)$ : In this case $\Pi_{K}=\emptyset$, so $R=R_{M}$. The set of positive $T$-roots coincides with $R^{+}$, which is connected. This corresponds to the space $G_{2} / T^{2}$. 
(2) $G_{2}\left(\alpha_{1}\right)$ : Here $\Pi_{K}=\left\{\alpha_{1}\right\}$, and $R_{M}^{+}=\left\{-\left(2 \varepsilon_{2}+\varepsilon_{3}\right),-\left(\varepsilon_{2}+2 \varepsilon_{3}\right), \varepsilon_{2}-\varepsilon_{3}\right.$, $\left.-\left(\varepsilon_{2}+\varepsilon_{3}\right),-\varepsilon_{3}\right\}$. Hence $\mathfrak{t}=\mathbb{R} \pi_{2}$, so $\operatorname{pr}_{\mathfrak{t}} \alpha_{2}=c \pi_{2}=\bar{\alpha}_{2}$. Therefore, each projection of $\alpha \in R_{M}^{+}$on $\mathfrak{t}$ is either $\bar{\alpha}_{2}$ or $2 \bar{\alpha}_{2}$, so $R_{T}^{+}=\left\{\bar{\alpha}_{2}, 2 \bar{\alpha}_{2}\right\}$, which is not connected. This corresponds to the space $G_{2} / U(2)$, with $U(2)$ represented by the long root of $G_{2}$.

(3) $G_{2}\left(\alpha_{2}\right)$ : Then $\Pi_{K}=\left\{\alpha_{2}\right\}, \mathfrak{t}=\mathbb{R} \pi_{1}$, and $\operatorname{pr}_{\mathfrak{t}} \alpha_{1}=c^{\prime} \pi_{1}=\bar{\alpha}_{1}$. By evaluating the projections of each $\alpha \in R_{M}^{+}$on $\mathfrak{t}$, we obtain that $R_{T}^{+}=\left\{\bar{\alpha}_{1}, 2 \bar{\alpha}_{1}, 3 \bar{\alpha}_{1}\right\}$, which is connected. This corresponds to the space $G_{2} / U(2)$, with $U(2)$ represented by the short root of $G_{2}$.

Case of $\mathbf{F}_{4}$. We fix the set of positive roots to be

$$
R^{+}=\left\{\varepsilon_{1}, \varepsilon_{2}, \varepsilon_{3}, \varepsilon_{4}, \varepsilon_{i} \pm \varepsilon_{j}(i<j), \frac{1}{2}\left(\varepsilon_{1} \pm \varepsilon_{2} \pm \varepsilon_{3} \pm \varepsilon_{4}\right)\right\} .
$$

The maximal root is expressed in terms of simple roots as $\mu=\varepsilon_{1}+\varepsilon_{2}=2 \alpha_{1}+4 \alpha_{2}+$ $3 \alpha_{3}+2 \alpha_{4}$, and any root $\alpha \in R$ is expressed as $\alpha=k_{1} \alpha_{1}+k_{2} \alpha_{2}+k_{3} \alpha_{3}+k_{4} \alpha_{4}$, with $\left|k_{1}\right| \leq 2,\left|k_{2}\right| \leq 4,\left|k_{3}\right| \leq 3,\left|k_{4}\right| \leq 2$. We denote such an expression by $\alpha=\left(k_{1}, k_{2}, k_{3}, k_{4}\right)$; hence each positive root is given as follows (see also [Fr-dV], Appendix Tables B and E]):

$$
\begin{aligned}
& \varepsilon_{1}=(2,3,2,1), \varepsilon_{2}=(0,1,1,1), \varepsilon_{3}=(0,1,1,0), \varepsilon_{4}=(0,1,0,0), \\
& \varepsilon_{1}-\varepsilon_{2}=(2,2,1,0), \varepsilon_{1}-\varepsilon_{3}=(2,2,1,1), \varepsilon_{1}-\varepsilon_{4}=(2,2,2,1), \varepsilon_{2}-\varepsilon_{3}=(0,0,0,1), \\
& \varepsilon_{2}-\varepsilon_{4}=(0,0,1,1), \varepsilon_{3}-\varepsilon_{4}=(0,0,1,0), \varepsilon_{1}+\varepsilon_{2}=(2,4,3,2), \varepsilon_{1}+\varepsilon_{3}=(2,4,3,1), \\
& \varepsilon_{1}+\varepsilon_{4}=(2,4,2,1), \varepsilon_{2}+\varepsilon_{3}=(0,2,2,1), \varepsilon_{2}+\varepsilon_{4}=(0,2,1,1), \varepsilon_{3}+\varepsilon_{4}=(0,2,1,0), \\
& \frac{1}{2}\left(\varepsilon_{1}+\varepsilon_{2}+\varepsilon_{3}+\varepsilon_{4}\right)=(1,3,2,1), \frac{1}{2}\left(\varepsilon_{1}+\varepsilon_{2}+\varepsilon_{3}-\varepsilon_{4}\right)=(1,2,2,1), \\
& \frac{1}{2}\left(\varepsilon_{1}+\varepsilon_{2}-\varepsilon_{3}+\varepsilon_{4}\right)=(1,2,1,1), \frac{1}{2}\left(\varepsilon_{1}-\varepsilon_{2}+\varepsilon_{3}+\varepsilon_{4}\right)=(1,2,1,0), \\
& \frac{1}{2}\left(\varepsilon_{1}+\varepsilon_{2}-\varepsilon_{3}-\varepsilon_{4}\right)=(1,1,1,1), \frac{1}{2}\left(\varepsilon_{1}-\varepsilon_{2}+\varepsilon_{3}-\varepsilon_{4}\right)=(1,1,1,0), \\
& \frac{1}{2}\left(\varepsilon_{1}-\varepsilon_{2}-\varepsilon_{3}+\varepsilon_{4}\right)=(1,1,0,0), \frac{1}{2}\left(\varepsilon_{1}-\varepsilon_{2}-\varepsilon_{3}-\varepsilon_{4}\right)=(1,0,0,0), \\
& 2 \varepsilon=(1,2,3,2,1,2) .
\end{aligned}
$$

(1) $F_{4}(0)$ : The set of positive $T$-roots coincides with $R^{+}$, which is connected. This corresponds to the case $F_{4} / T^{4}$.

(2) $F_{4}\left(\alpha_{2}, \alpha_{3}, \alpha_{4}\right)$ : Then $\mathfrak{t}=\mathbb{R} \pi_{1}$ and $\operatorname{pr}_{\mathfrak{t}} \alpha=k_{1} \bar{\alpha}_{1}$ for each $\alpha \in R_{M}^{+}$(recall that $\operatorname{pr}_{\mathfrak{t}} \alpha=0$ if $\left.\alpha \in R_{K}\right)$. We obtain that $R_{T}^{+}=\left\{\bar{\alpha}_{1}, 2 \bar{\alpha}_{1}\right\}$, which is not connected. This corresponds to the space $F_{4} / S O(7) \cdot U(1)$.

(3) $F_{4}\left(\alpha_{1}, \alpha_{2}, \alpha_{3}\right)$ : Then $\mathfrak{t}=\mathbb{R} \pi_{4}$ and $\operatorname{pr}_{\mathfrak{t}} \alpha=k_{4} \bar{\alpha}_{4}$. It follows that $R_{T}^{+}=$ $\left\{\bar{\alpha}_{4}, 2 \bar{\alpha}_{4}\right\}$, which is not connected. This corresponds to the space $F_{4} / S p(3) \cdot U(1)$.

(4) $F_{4}\left(\alpha_{1}, \alpha_{2}\right)$ : Here $\mathfrak{t}=\operatorname{span}_{\mathbb{R}}\left(\pi_{3}, \pi_{4}\right)$ and $\operatorname{pr}_{\mathfrak{t}} \alpha=k_{3} \bar{\alpha}_{3}+k_{4} \bar{\alpha}_{4}$ for each $\alpha \in R_{M}^{+}$. We obtain the system of positive $T$-roots $R_{T}^{+}=\left\{2 \bar{\alpha}_{3}+\bar{\alpha}_{4}, \bar{\alpha}_{3}+\bar{\alpha}_{4}, \bar{\alpha}_{3}, \bar{\alpha}_{4}, 3 \bar{\alpha}_{3}+\right.$ $\left.2 \bar{\alpha}_{4}, 3 \bar{\alpha}_{3}+\bar{\alpha}_{4}\right\}$, which is connected.

(5) $F_{4}\left(\alpha_{1}, \alpha_{3}, \alpha_{4}\right)$ : Here $\mathfrak{t}=\mathbb{R} \pi_{2}$ and $\operatorname{pr}_{\mathfrak{t}} \alpha=k_{2} \bar{\alpha}_{2}$. We obtain that $R_{T}^{+}=$ $\left\{\bar{\alpha}_{2}, 2 \bar{\alpha}_{2}, 3 \bar{\alpha}_{2}, 4 \bar{\alpha}_{2}\right\}$, which is connected.

In all other cases the set of $T$-roots is also connected.

Case of $\mathbf{E}_{\mathbf{6}}$. We fix the set of positive roots to be

$$
R^{+}=\left\{\varepsilon_{i}-\varepsilon_{j}(i<j), 2 \varepsilon, \varepsilon_{i}+\varepsilon_{j}+\varepsilon_{k}+\varepsilon(i<j<k)\right\} .
$$


The maximal root is expressed as $\mu=2 \varepsilon=\alpha_{1}+2 \alpha_{2}+3 \alpha_{3}+2 \alpha_{4}+\alpha_{5}+2 \alpha_{6}$. By using the notation as in $F_{4}$ each positive root is given as follows:

$$
\begin{aligned}
& \varepsilon_{1}-\varepsilon_{2}=(1,0,0,0,0,0), \varepsilon_{2}-\varepsilon_{3}=(0,1,0,0,0,0), \varepsilon_{3}-\varepsilon_{4}=(0,0,1,0,0,0), \\
& \varepsilon_{4}-\varepsilon_{5}=(0,0,0,1,0,0), \varepsilon_{5}-\varepsilon_{6}=(0,0,0,0,1,0), \varepsilon_{1}-\varepsilon_{3}=(1,1,0,0,0,0), \\
& \varepsilon_{2}-\varepsilon_{4}=(0,1,1,0,0,0), \varepsilon_{3}-\varepsilon_{5}=(0,0,1,1,0,0), \varepsilon_{4}-\varepsilon_{6}=(0,0,0,1,1,0), \\
& \varepsilon_{1}-\varepsilon_{4}=(1,1,1,0,0,0), \varepsilon_{1}-\varepsilon_{5}=(1,1,1,1,0,0), \varepsilon_{1}-\varepsilon_{6}=(1,1,1,1,1,0), \\
& \varepsilon_{2}-\varepsilon_{5}=(0,1,1,1,0,0), \varepsilon_{2}-\varepsilon_{6}=(0,1,1,1,1,0), \varepsilon_{3}-\varepsilon_{6}=(0,0,1,1,1,0), \\
& \varepsilon_{1}+\varepsilon_{2}+\varepsilon_{3}+\varepsilon=(1,2,3,2,1,1), \varepsilon_{1}+\varepsilon_{2}+\varepsilon_{4}+\varepsilon=(1,2,2,2,1,1), \\
& \varepsilon_{1}+\varepsilon_{2}+\varepsilon_{5}+\varepsilon=(1,2,2,1,1,1), \varepsilon_{1}+\varepsilon_{2}+\varepsilon_{6}+\varepsilon=(1,2,2,1,0,1), \\
& \varepsilon_{1}+\varepsilon_{3}+\varepsilon_{4}+\varepsilon=(1,1,2,2,1,1), \varepsilon_{1}+\varepsilon_{3}+\varepsilon_{5}+\varepsilon=(1,1,2,1,1,1), \\
& \varepsilon_{1}+\varepsilon_{3}+\varepsilon_{6}+\varepsilon=(1,1,2,1,0,1), \varepsilon_{1}+\varepsilon_{4}+\varepsilon_{5}+\varepsilon=(1,1,1,1,1,1), \\
& \varepsilon_{1}+\varepsilon_{4}+\varepsilon_{6}+\varepsilon=(1,1,1,1,0,1), \varepsilon_{1}+\varepsilon_{5}+\varepsilon_{6}+\varepsilon=(1,1,1,0,0,1), \\
& \varepsilon_{2}+\varepsilon_{3}+\varepsilon_{4}+\varepsilon=(0,1,2,2,1,1), \varepsilon_{2}+\varepsilon_{3}+\varepsilon_{5}+\varepsilon=(0,1,2,1,1,1), \\
& \varepsilon_{2}+\varepsilon_{3}+\varepsilon_{6}+\varepsilon=(0,1,2,1,0,1), \varepsilon_{2}+\varepsilon_{4}+\varepsilon_{5}+\varepsilon=(0,1,1,1,1,1), \\
& \varepsilon_{2}+\varepsilon_{4}+\varepsilon_{6}+\varepsilon=(0,1,1,1,0,1), \varepsilon_{2}+\varepsilon_{5}+\varepsilon_{6}+\varepsilon=(0,1,1,0,0,1), \\
& \varepsilon_{3}+\varepsilon_{4}+\varepsilon_{5}+\varepsilon=(0,0,1,1,1,1), \varepsilon_{3}+\varepsilon_{4}+\varepsilon_{6}+\varepsilon=(0,0,1,1,0,1), \\
& \varepsilon_{3}+\varepsilon_{5}+\varepsilon_{6}+\varepsilon=(0,0,1,0,0,1), \varepsilon_{4}+\varepsilon_{5}+\varepsilon_{6}+\varepsilon=(0,0,0,0,0,1),
\end{aligned}
$$

(1) $E_{6}\left(\alpha_{1}, \alpha_{2}, \alpha_{3}, \alpha_{4}, \alpha_{5}\right): \mathfrak{t}=\mathbb{R} \pi_{6}$ and $\operatorname{pr}_{\mathfrak{t}} \alpha=k_{6} \bar{\alpha}_{6}$ for each $\alpha \in R_{M}^{+}$. The set of positive $T$-roots is $R_{T}^{+}=\left\{\bar{\alpha}_{6}, 2 \bar{\alpha}_{6}\right\}$, which is not connected. This corresponds to the space $E_{6} / S U(6) \cdot U(1)$.

(2) $E_{6}\left(\alpha_{1}, \alpha_{3}, \alpha_{4}, \alpha_{5}, \alpha_{6}\right): \mathfrak{t}=\mathbb{R} \pi_{2}$ and $\operatorname{pr}_{\mathfrak{t}} \alpha=k_{2} \bar{\alpha}_{2}$. The set of positive $T$ roots is $R_{T}^{+}=\left\{\bar{\alpha}_{2}, 2 \bar{\alpha}_{2}\right\}$, which is not connected. This corresponds to the space $E_{6} / S U(2) \cdot S U(5) \cdot U(1)$.

In all other cases the set of $T$-roots is connected. We show two cases:

(3) $E_{6}\left(\alpha_{3}, \alpha_{4}, \alpha_{5}\right): \mathfrak{t}=\operatorname{span}_{\mathbb{R}}\left(\pi_{1}, \pi_{2}, \pi_{6}\right)$ and $\operatorname{pr}_{\mathfrak{t}} \alpha=k_{1} \bar{\alpha}_{1}+k_{2} \bar{\alpha}_{2}+k_{6} \bar{\alpha}_{6}$. We obtain that $R_{T}^{+}=\left\{\bar{\alpha}_{1}, \bar{\alpha}_{2}, \bar{\alpha}_{1}+\bar{\alpha}_{2}, \bar{\alpha}_{1}+2 \bar{\alpha}_{2}+\bar{\alpha}_{6}, \bar{\alpha}_{1}+\bar{\alpha}_{2}+\bar{\alpha}_{6}, \bar{\alpha}_{2}+\bar{\alpha}_{6}, \bar{\alpha}_{6}, \bar{\alpha}_{1}+\right.$ $\left.2 \bar{\alpha}_{2}+2 \bar{\alpha}_{6}\right\}$, which is connected.

(4) $E_{6}\left(\alpha_{1}, \alpha_{2}, \alpha_{4}, \alpha_{5}\right): \mathfrak{t}=\operatorname{span}_{\mathbb{R}}\left(\pi_{3}, \pi_{6}\right)$ and $\operatorname{pr}_{\mathfrak{t}} \alpha=k_{3} \bar{\alpha}_{3}+k_{6} \bar{\alpha}_{6}$. We obtain that $R_{T}^{+}=\left\{\bar{\alpha}_{3}, 3 \bar{\alpha}_{3}+\bar{\alpha}_{6}, 2 \bar{\alpha}_{3}+\bar{\alpha}_{6}, \bar{\alpha}_{3}+\bar{\alpha}_{6}, \bar{\alpha}_{6}\right\}$, which is connected.

Case of $\mathbf{E}_{\mathbf{7}}$. We fix the set of positive roots to be

$$
\begin{aligned}
R^{+}= & \left\{\varepsilon_{i}-\varepsilon_{j}(i<j \leq 7),-\left(\varepsilon_{i}-\varepsilon_{8}\right),-\left(\varepsilon_{i}+\varepsilon_{j}+\varepsilon_{k}+\varepsilon_{l}\right)(i<j<k<l \leq 7),\right. \\
& \left.\varepsilon_{i}+\varepsilon_{j}+\varepsilon_{k}+\varepsilon_{8}(i<j<k)\right\} .
\end{aligned}
$$

The maximal root is given as $\mu=-\varepsilon_{7}+\varepsilon_{8}=\alpha_{1}+2 \alpha_{2}+3 \alpha_{3}+4 \alpha_{4}+3 \alpha_{5}+2 \alpha_{6}+2 \alpha_{7}$.

The set of $T$-roots is not connected only in the following cases :

(1) $E_{7}\left(\alpha_{1}, \alpha_{2}, \alpha_{3}, \alpha_{4}, \alpha_{5}, \alpha_{6}\right)$ : We obtain that $\mathfrak{t}=\mathbb{R} \pi_{7}$ and $\operatorname{pr}_{\mathfrak{t}} \alpha=k_{7} \bar{\alpha}_{7}$. The set of positive $T$-roots is $R_{T}^{+}=\left\{\bar{\alpha}_{7}, 2 \bar{\alpha}_{7}\right\}$, which is not connected. This corresponds to the space $E_{7} / S U(7) \cdot U(1)$.

(2) $E_{7}\left(\alpha_{1}, \alpha_{3}, \alpha_{4}, \alpha_{5}, \alpha_{6}, \alpha_{7}\right)$ : We have that $\mathfrak{t}=\mathbb{R} \pi_{2}$ and $\operatorname{pr}_{\mathfrak{t}} \alpha=k_{2} \bar{\alpha}_{2}$. The set of positive $T$-roots is $R_{T}^{+}=\left\{\bar{\alpha}_{2}, 2 \bar{\alpha}_{2}\right\}$, which is not connected. This corresponds to the space $E_{7} / S U(2) \cdot S O(10) \cdot U(1)$. 
(3) $E_{7}\left(\alpha_{1}, \alpha_{2}, \alpha_{3}, \alpha_{4}, \alpha_{5}, \alpha_{7}\right):$ Here $\mathfrak{t}=\mathbb{R} \pi_{6}$ and $\operatorname{pr}_{\mathfrak{t}} \alpha=k_{6} \bar{\alpha}_{6}$. We obtain that $R_{T}^{+}=\left\{\bar{\alpha}_{6}, 2 \bar{\alpha}_{6}\right\}$, which is not connected. This corresponds to the space $E_{7} / S O(12)$. $U(1)$.

Case of $\mathbf{E}_{\mathbf{8}}$. We fix the set of positive roots to be

$$
R^{+}=\left\{\varepsilon_{i}-\varepsilon_{j}(i<j), \varepsilon_{i}+\varepsilon_{j}+\varepsilon_{k}(i<j<k \leq 8),-\left(\varepsilon_{i}+\varepsilon_{j}+\varepsilon_{9}\right)(i<j)\right\} .
$$

The maximal root is expressed as $\mu=\varepsilon_{1}-\varepsilon_{9}=2 \alpha_{1}+3 \alpha_{2}+4 \alpha_{3}+5 \alpha_{4}+6 \alpha_{5}+$ $4 \alpha_{6}+2 \alpha_{7}+3 \alpha_{8}$.

The set of $T$-roots is not connected only in the following cases:

(1) $E_{8}\left(\alpha_{2}, \alpha_{3} \ldots, \alpha_{8}\right)$ : Here $\mathfrak{t}=\mathbb{R} \pi_{1}$ and $\operatorname{pr}_{\mathfrak{t}} \alpha=k_{1} \bar{\alpha}_{1}$. A computation gives that $R_{T}^{+}=\left\{\bar{\alpha}_{1}, 2 \bar{\alpha}_{1}\right\}$, which is not connected. This corresponds to the space $E_{8} / E_{7} \cdot U(1)$.

(2) $E_{8}\left(\alpha_{1}, \ldots, \alpha_{6}, \alpha_{8}\right):$ Here $\mathfrak{t}=\mathbb{R} \pi_{7}$ and $\operatorname{pr}_{\mathfrak{t}} \alpha=k_{7} \bar{\alpha}_{7}$. We obtain that $R_{T}^{+}=$ $\left\{\bar{\alpha}_{7}, 2 \bar{\alpha}_{7}\right\}$, which is not connected. This corresponds to the space $E_{8} / S O(14) \cdot U(1)$.

Summarizing these results, we get the following.

Proposition 10. Let $M=G / K$ be a flag manifold of an exceptional Lie group $G$. If the $T$-root system $R_{T}$ is not connected, then it has the form $R_{T}=\{ \pm \xi, \pm 2 \xi\}$ and the manifold $M$ is equivalent to one of the following flag manifolds:

$$
\begin{aligned}
& G_{2}\left(\alpha_{1}\right)=G_{2} / U(2) \quad \begin{array}{cr}
\alpha_{1} & \alpha_{2} \\
\hline & \Longrightarrow \bullet
\end{array} \\
& F_{4}\left(\alpha_{2}, \alpha_{3}, \alpha_{4}\right)=F_{4} / S O(7) \cdot U(1) \quad \begin{array}{ccccc}
\alpha_{1} & \alpha_{2} & \alpha_{3} & \alpha_{4} \\
\alpha_{1} & \alpha_{2} & \alpha_{3} & \alpha_{4}
\end{array} \\
& F_{4}\left(\alpha_{1}, \alpha_{2}, \alpha_{3}\right)=F_{4} / S p(3) \cdot U(1) \\
& E_{6}\left(\alpha_{1}, \alpha_{2}, \alpha_{3}, \alpha_{4}, \alpha_{5}\right)=E_{6} / S U(6) \cdot U(1) \\
& \begin{array}{lllll}
\alpha_{1} & \alpha_{2} & \alpha_{3} & \alpha_{4} & \alpha_{5}
\end{array} \\
& E_{6}\left(\alpha_{1}, \alpha_{3}, \alpha_{4}, \alpha_{5}, \alpha_{6}\right)=E_{6} / S U(2) \cdot S U(5) \cdot U(1) \\
& \alpha_{1} \quad \alpha_{2} \quad \alpha_{3} \quad \alpha_{4} \quad \alpha_{5} \\
& \text { ○ } \\
& E_{7}\left(\alpha_{1}, \alpha_{2}, \alpha_{3}, \alpha_{4}, \alpha_{5}, \alpha_{6}\right)=E_{7} / S U(7) \cdot U(1) \\
& \begin{array}{llllll}
\alpha_{1} & \alpha_{2} & \alpha_{3} & \alpha_{4} & \alpha_{5} & \alpha_{6}
\end{array} \\
& E_{7}\left(\alpha_{1}, \alpha_{2}, \alpha_{3}, \alpha_{4}, \alpha_{6}, \alpha_{7}\right)=E_{7} / S U(2) \cdot S O(10) \cdot U(1) \\
& E_{7}\left(\alpha_{1}, \alpha_{3}, \alpha_{4}, \alpha_{5}, \alpha_{5}, \alpha_{7}\right)=E_{7} / S O(12) \cdot U(1) \\
& \begin{array}{llllll}
\alpha_{1} & \alpha_{2} & \alpha_{3} & \alpha_{4} & \alpha_{5} & \alpha_{6}
\end{array} \\
& E_{8}\left(\alpha_{2}, \alpha_{3}, \alpha_{4}, \alpha_{5}, \alpha_{6}, \alpha_{7}, \alpha_{8}\right)=E_{8} / E_{7} \cdot U(1) \\
& E_{8}\left(\alpha_{1}, \alpha_{2}, \alpha_{3}, \alpha_{4}, \alpha_{5}, \alpha_{6}, \alpha_{8}\right)=E_{8} / S O(14) \cdot U(1)
\end{aligned}
$$
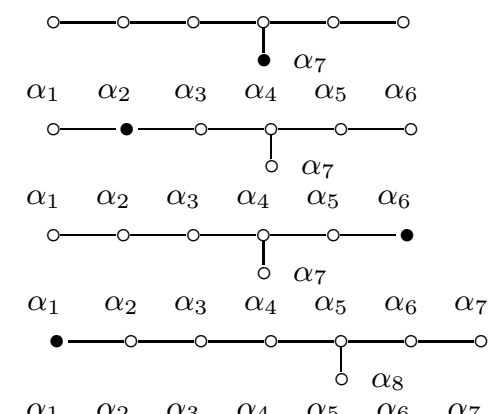

Corollary 11. Let $M=G / K$ be a flag manifold of a simple Lie group $G$. Then the $T$-root system $R_{T}$ is not connected if and only if it has the form $R_{T}=\{ \pm \xi, \pm 2 \xi\}$ for some 1-form $\xi$ on $\mathfrak{t}$ or, equivalently, the isotropy representation of $K$ has two irreducible components.

We will give another characterization of the flag manifolds with non-connected $R_{T}$, described above. 
According to Section 3, T-roots $\xi \in R_{T}$ of a flag manifold $M=G / K$ correspond to irreducible $K$-submodules $\mathfrak{m}_{\xi}$ of the complexified tangent space $\left(T_{o} M\right)^{C}=\mathfrak{m}^{\mathbb{C}}$ of $M$. In particular, a flag manifold $M=G / K$ with the $T$-root system of the form $R_{T}=\{ \pm \xi, \pm 2 \xi\}$ defines a depth-two gradation

$$
\mathfrak{g}^{\mathbb{C}}=\mathfrak{g}_{-2}+\mathfrak{g}_{-1}+\mathfrak{g}_{0}+\mathfrak{g}_{1}+\mathfrak{g}_{2}, \quad\left[\mathfrak{g}_{i}, \mathfrak{g}_{j}\right] \subset \mathfrak{g}_{i+j}
$$

of the complexification $\mathfrak{g}^{\mathbb{C}}$ of the Lie algebra $\mathfrak{g}$ with irreducible $K$-module $\mathfrak{m}_{-1}$, given by

$$
\mathfrak{g}_{0}=\mathfrak{k}^{\mathbb{C}}, \mathfrak{g}_{i}=\mathfrak{m}_{i \xi}, \quad i= \pm 1, \pm 2
$$

Moreover, we have a 1-1 correspondence between such flag manifolds and depthtwo gradations with irreducible modules $\mathfrak{g}_{i}$. Such gradations of a complex simple Lie algebra $\mathfrak{g}^{\mathbb{C}}$ correspond to simple roots $\alpha$ of a simple root system $\Pi$ of the Lie algebra $\mathfrak{g}^{\mathbb{C}}$ (with respect to a Cartan subalgebra $\mathfrak{h}^{\mathbb{C}}$ ), with Dynkin mark 2 (cf. Gor-On-Vin]). Recall that the Dynkin marks $n_{i}$ (also known as "heights" of the roots) are the coefficients (positive integers) of the decomposition of the maximal root $\mu$ with respect to the simple roots $\alpha_{i} \in \Pi: \mu=\sum n_{i} \alpha_{i}$.

The gradation associated to a simple root $\alpha$ is defined by the conditions

$$
\operatorname{deg} \mathfrak{h}=\operatorname{deg} E_{\beta}=0, \text { for all } \beta \in \Pi \backslash\{\alpha\}, \operatorname{deg} E_{\alpha}=1 \text {. }
$$

The associated flag manifold is $M_{\alpha}=G / K_{\alpha}$, where $K_{\alpha}$ is the compact subgroup of $G$ which corresponds to the Lie subalgebra $\mathfrak{k}^{\mathbb{C}}$ of $\mathfrak{g}^{\mathbb{C}}$ generated by the Cartan subalgebra $\mathfrak{h}^{\mathbb{C}}$ and the root vectors $E_{\beta}, \beta \in \Pi \backslash\{\alpha\}$.

Therefore we obtain:

Corollary 12. The flag manifolds $M=G / K$ of a simple Lie group $G$ with nonconnected $R_{T}$ are exactly the flag manifolds $M_{\alpha}=G / K_{\alpha}$ associated with a simple root $\alpha$ with Dynkin mark 2.

\section{Flag manifolds With homogeneous Geodesics}

In this section we describe flag manifolds $M=G / K$ with non-connected $T$-root system $R_{T}$, and which have non-standard metrics with homogeneous geodesics. By Corollary 12 any such manifold $M=M_{\alpha}$ is associated to a simple root $\alpha$ of the complex Lie algebra $\mathfrak{g}^{\mathbb{C}}$ and defines a depth-two gradation

$$
\mathfrak{g}^{\mathbb{C}}=\mathfrak{g}_{-2}+\mathfrak{g}_{-1}+\mathfrak{g}_{0}+\mathfrak{g}_{1}+\mathfrak{g}_{2},
$$

where $\mathfrak{g}_{0}=\mathfrak{k}^{\mathbb{C}}$, with irreducible $K$-module $\mathfrak{g}_{-1}$.

The even and odd parts of this gradation determine a symmetric decomposition

$$
\mathfrak{g}^{\mathbb{C}}=\mathfrak{g}_{\mathrm{e} v}+\mathfrak{g}_{\mathrm{odd}}=\left(\mathfrak{g}_{-2}+\mathfrak{g}_{0}+\mathfrak{g}_{2}\right)+\left(\mathfrak{g}_{-1}+\mathfrak{g}_{1}\right)
$$

of the Lie algebra $\mathfrak{g}^{\mathbb{C}}$. We denote by $G / G_{\mathrm{e} v}$ the corresponding compact symmetric space, where $G_{\mathrm{e} v}$ is the compact subgroup of $G$ which corresponds to the subalgebra $\mathfrak{g}_{\mathrm{e} v}$. Consider the natural fibration

$$
\pi: M_{\alpha}=G / K \rightarrow G / G_{\mathrm{e} v}
$$

with fiber $F=G_{\mathrm{e} v} / K$. Since $F$ is an isotropy irreducible homogeneous space (whose tangent space can be identified with $\mathfrak{n}:=\mathfrak{g} \cap\left(\mathfrak{g}_{-1}+\mathfrak{g}_{1}\right)$ ), any invariant metric on $M_{\alpha}$ is homothetic to the metric $g_{\lambda}$, which is obtained from the standard metric $g_{1}$ by multiplying its $\pi$-vertical part $\left.g_{1}\right|_{\mathfrak{n}}$ by a positive constant $\lambda$.

In the paper [Ta], H. Tamaru classified all such metrics $g_{\lambda}$ with homogeneous geodesics on homogeneous manifolds $G / K$ of a compact simple Lie group $G$ which 
are fibered over a symmetric space $G / H$. Combining his classification with our results we conclude that the only flag manifolds $M=G / K$ of a simple Lie group which admit non-standard metrics $g_{\lambda}(\lambda \neq 1)$ with homogeneous geodesics are the manifolds $B(\ell, 0)=S O(2 \ell+1) / U(\ell)$ and $C(1, \ell-1)=S p(\ell) / U(1) \cdot S p(\ell-1)$.

Also, in $\mathrm{On}$, A.L. Onishchik proved that the only compact connected enlargenent of the transformation group $S O(2 \ell+1)$ of $B(\ell, 0)$ is $S O(2 \ell+2)$, and the only compact connected enlargement of the transformation group $S p(\ell)$ of $C(1, \ell-1)$ is the group $S U(2 \ell-1)$. In addition, $B(\ell, 0)=S O(2 \ell+1) / U(\ell)=S O(2 \ell+2) / U(\ell+1)$ and $C(1, \ell-1)=S p(\ell) / U(1) \cdot S p(\ell-1)=S U(2 \ell-1) / U(2 \ell-2)$. Due to this, the manifold $B(\ell, 0)$ is identified with the symmetric manifold $\operatorname{Com}\left(\mathbb{R}^{2 \ell+2}\right)$ of complex structures in $\mathbb{R}^{2 \ell+2}$, and the manifold $C(1, \ell-1)$ is identified with the projective space $\mathbb{C} P^{2 \ell-2}$. The metric $g_{1}$ gives the standard symmetric metric on these manifolds. Any other metric $g_{\lambda}, \lambda \neq 1$ cannot be invariant under the extended group. Therefore, the full connected groups of isometries of these spaces $B(\ell, 0)$ and $C(1, \ell-1)$ are $S O(2 \ell+1)$ and $S p(\ell)$, respectively. This proves Theorem III stated in the introduction.

\section{Acknowledgement}

The authors would like to thank Professor Oldrich Kowalski for several useful discussions and Professor Yusuke Sakane for bringing to their attention reference Ta.

\section{REFERENCES}

[A] D.V. Alekseevsky: Flag manifolds, in: Sbornik Radova, 11 Jugoslav. Seminr. Beograd 6(14) (1997) 3-35. MR1491979 (99b:53073)

[A-Ar] D.V. Alekseevsky - A. Arvanitoyeorgos: Metrics with homogeneous geodesics on flag manifolds, Comment. Math. Univ. Carolinae 43 (2) (2002) 189-199. MR.1922121 (2003g:53079)

[A-P] D.V. Alekseevsky - A.M. Perelomov: Invariant Kähler-Einstein metrics on compact homogeneous spaces, Funct. Anal. Applic., 20 (1986) 171-182.

[Ak-Vin] D.N. Akhiezer - E.B. Vinberg: Weakly symmetric spaces and spherical varieties, Transform. Groups 4 (1999) 3-24. MR:1669186 (2000b:14064)

[Arn] V.I. Arnold: Mathematical Methods of Classical Mechanics, Springer-Verlag, 1978. MR0690288 (57:14033b)

[Be] A. Besse: Einstein Manifolds, Springer-Verlag, Berlin, 1987. MR0867684 (88f:53087)

[B-F-R] M. Bordemann - M. Forger - H. Römer: Homogeneous Kähler manifolds: Paving the way towards new supersymmetric sigma models, Comm. Math. Phys. 102 (1986) 605-647. MR0824094 (87c:53096)

[Bour] N. Bourbaki: Elements of Mathematics. Lie Groups and Lie Algebras, SpringerVerlag, New York, 2002. MR1890629 (2003a:17001)

[DA-Zi] J.E. D'Atri - W. Ziller: Naturally reductive metrics and Einstein metrics on compact Lie groups, Memoirs Amer. Math. Soc. 18 (215) (1979). MR0519928 (80i:53023)

[Du1] Z. Dušek: Structure of geodesics in a 13-dimensional group of Heisenberg type, Proc. Coll. Diff. Geom. in Debrecen (2001) 95-103. MR.1859291 (2002f:53057)

[Du2] Z. Dušek: Explicit geodesic graphs on some H-type groups, Rend. Circ. Mat. Palermo Ser. II, Suppl. 69 (2002) 77-88. MR.1972426 (2004b:53071)

[Du-Ko-Ni] Z. Dušek - O. Kowalski - S. Ž. Nikčević: New examples of g.o. spaces in dimension 7, Diff. Geom. Appl. 21 (2004) 65-78. MR2067459 (2005b:53081)

[Fr-dV] H. Freudenthal - H. de Vries: Linear Lie Groups, Academic Press, New York, 1969. MR0260926 (41:5546) 
[Ga-Hu-Wi] L. Gagnon - V. Hussin - P. Winternitz: Nonlinear equations with superposition formulas and exceptional group $G_{2}$. III. The superposition formulas, J. Math. Phys. 29 (10) (1988) 2145-2155. MR0962548 (89j:58117)

[Go] C. S. Gordon: Homogeneous manifolds whose geodesics are orbits, in: Topics in Geometry, in Memory of Joseph D'Atri, Birkhäuser, Basel, 1996, 155-174. MR1390313 (97d:53055)

[Gor-On-Vin] V.V. Gorbatzevich - A.L. Onishchik - E.B. Vinberg: Structure of Lie Groups and Lie Algebras, Encycl. of Math. Sci. v41, Lie Groups and Lie Algebras-3, SpringerVerlag. MR1349140(96d:22001)

[Ka] A. Kaplan: On the geometry of groups of Heisenberg type, Bull. London Math. Soc. 15 (1983) 35-42. MR0686346 (84h:53063)

[Kaj] V.V. Kajzer: Conjugate points of left-invariant metrics on Lie Groups, J. Soviet Math. 34 (1990) 32-44. MR.1106314 (92d:53042)

[Kos1] B. Kostant: Holonomy and Lie algebra of motions in Riemannian manifolds, Trans. AMS, 80 (1955) 520-542. MR0084825(18:930a)

[Kos2] B. Kostant: On differential geometry and homogeneous spaces II, Proc. N.A.S. U.S.A. 42 (1956) 354-357. MR0088017 (19:454a)

[Ko-Ni] O. Kowalski - S. Ž. Nikčević: On geodesic graphs of Riemannian g.o. spaces, Arch. Math. 73 (1999) 223-234. MR1705019 (2000e:53062)

[Ko-Ni-Vl] O. Kowalski - S. Ž. Nikčević - Z. Vlašek: Homogeneous geodesics in homogeneous Riemannian manifolds - examples, in: Geometry and Topology of Submanifolds, X (Beijing/Berlin, 1999), 104-112, World Sci. Publishing, River Edge, NJ, 2000. MR:1801906 (2001j:53050)

[Ko-Va] O. Kowalski - L. Vanhecke: Riemannian manifolds with homogeneous geodesics, Boll. Un. Math. Ital. B (7) 5 (1991) 189-246. MR.1110676 (92m:53084)

[Ko-Sz] O. Kowalski - J. Szenthe: On the existence of homogeneous geodesics in homogeneous Riemannian manifolds, Geom. Ded. 81 (2000) 209-214, Erratum: 84 (2001) 331-332. MR.1772203 (2001f:53104)

[Ko-Pr-Va] O. Kowalski - F. Prüfer - L. Vanhecke: D'Atri spaces, in: Topics in Geometry: In Memory of Joseph D'Atri, Birkhäuser (1996) 240-284. MR1390318 (97m:53086)

[Ma] R.A. Marinosci: Homogeneous geodesics in a three-dimensional Lie group, Comment. Math. Univ. Carolinae 43(2) (2002) 261-270. MR.1922126 (2003g:53052)

[On] A.L. Onishchik: Topology of Transitive Transformation Groups, Johann Ambrosius Barth, Leipzig-Heidelberg-Berlin, 1994. MR1379333 (97j:57057)

[Po-Th] F. Podestà - G. Thorbergsson: Coisotropic actions on compact homogeneous Kähler manifolds, Math. Z. 243 (2003) 471-490. MR1970013 (2004d:53060)

[Sa] H. Samelson: Notes on Lie Algebras, Springer-Verlag, New York, 1990. MR1056083 (91h:17006)

[Sz] J. Szenthe: Homogeneous geodesics of left-invariant metrics, Univ. Iagellonicae Acta Math. Fasc. XXXVIII (2000) 99-103. MR1812104 (2002f:53060)

[Sie] J. Siebenthal: Sur certains modules dans une algèbre de Lie semisimple, Comment. Math. Helv. 44(1) (1964) 1-44. MR0241488 (39:2828)

[Ta] H. Tamaru: Riemannian g.o. spaces fibered over irreducible symmetric spaces, Osaka J. Math. 36 (1999) 835-851. MR1745654 (2000m:53070)

[Vin] E.B. Vinberg: Invariant linear connections in a homogeneous manifold, Trudy MMO 9 (1960) 191-210. MR0176418 (31:690)

[Zi] W. Ziller: Weakly symmetric spaces, in: Topics in Geometry, in Memory of Joseph D'Atri, Birkhäuser, Basel, 1996, 355-368. MR 1390324 (97c:53081)

School of Mathematics and Maxwell Institute for Mathematical Studies, Edinburgh University, Edinburgh EH9 3JZ, United Kingdom

E-mail address: D.Aleksee@ed.ac.uk

Department of Mathematics, University of Patras, GR-26500 Patras, Greece

E-mail address: arvanito@math.upatras.gr 